\title{
RESTRICTIONS OF THE LAPLACE-BELTRAMI EIGENFUNCTIONS TO SUBMANIFOLDS
}

\author{
by
}

N. Burq, P. Gérard \& N. Tzvetkov

\begin{abstract}
We estimates the $L^{p}$ norm $(2 \leq p \leq+\infty)$ of the restriction to a curve of the eigenfunctions of the Laplace Beltrami operator on a riemannian surface. If the curve is a geodesic, we show that on the sphere these estimates are sharp. If the curve has non vanishing geodesic curvature, we can improve our results. All our estimates are shown to be optimal for the sphere. Moreover, we sketch their extension to higher dimension

Résumé. - On prouve une estimation de la norme $L^{p}(2 \leq p \leq+\infty)$ de la restriction à une courbe des fonctions propres de l'opérateur de Laplace Beltrami sur une surface riemannienne. Si la courbe est une géodésique de la sphère, on montre que nos estimations sont optimales. En revanche, si la courbe possède une courbure géodésique non nulle, on améliore le résultat. Toutes nos estimées sont optimales sur la sphère. Nous en esquissons par ailleurs des généralisations aux dimensions supérieures.
\end{abstract}

\section{Introduction}

Let $(M, g)$ be a compact smooth Riemannian manifold (without boundary) of dimension $d$. Let us denote by $\boldsymbol{\Delta}$ the Laplace operator associated to the metric $g$. Let $\Sigma:[a, b]^{k} \rightarrow M$ be a smooth embedded sub-manifold of dimension $k$. The metric $g$ endows $M$ and $\Sigma$ with canonical measures and consequently we can define the Lebesgue spaces $L^{p}(M)$ and $L^{p}(\Sigma), 1 \leq p \leq+\infty$, of functions on $M$ and $\Sigma$ respectively. Let $\left(\varphi_{\lambda}\right), \lambda \geq 0$, be the eigenfunctions of $\boldsymbol{\Delta}$ such that $-\boldsymbol{\Delta} \varphi_{\lambda}=$ $\lambda^{2} \varphi_{\lambda}$. This paper fits in the line of researches dealing with possible concentrations of the eigenfunctions of the Laplace operator on a manifold. There are many ways

2000 Mathematics Subject Classification. - 35P20, 35J15, 53C21.

Key words and phrases. — eigenfunction estimates. 
of measuring possible concentrations. One of the most popular is by describing semi-classical (Wigner) measures (see the works by Shnirelman [17, Zelditch [25, Colin de Verdière [5, Gérard-Leichtnam [7, Zelditch-Zworski 26], Helffer-MartinezRobert 9], Sarnak 16, Lindenstrauss 13, and Anantharaman 1]). Another way is the study of the potential growth of $\left\|\varphi_{\lambda}\right\|_{L^{p}(M)}$, see the works by Sogge [18, 19], Sogge-Zelditch [20, the authors [3, 2, 4]. In the present paper we propose a third way (see also the work by Reznikov [15) and study the possible growth of the $L^{p}$ norm $(2 \leq p \leq+\infty)$ of the restrictions of $\varphi_{\lambda}$ to submanifolds of $M$. In most of this article we will concentrate to the simplest case of curves on a surface (i.e., the case when $\Sigma$ is a smooth curve $\gamma:[a, b] \longrightarrow M$ parametrized by arc length. Our first result reads as follows.

Theorem 1. - There exists a constant $C$ such that for every $\varphi_{\lambda}$,

$$
\left\|\varphi_{\lambda}\right\|_{L^{p}(\gamma)} \leq C(1+\lambda)^{\delta(p)}\left\|\varphi_{\lambda}\right\|_{L^{2}(M)},
$$

where

$$
\delta(p)= \begin{cases}\frac{1}{2}-\frac{1}{p} & \text { if } 4 \leq p \leq+\infty \\ \frac{1}{4} & \text { if } 2 \leq p \leq 4 .\end{cases}
$$

Moreover (1.1) is sharp if $M$ is the standard sphere $\mathbb{S}^{2}$ when $\gamma$ is any curve for $4 \leq p \leq+\infty$ and when $\gamma$ is a geodesic curve for $2 \leq p<4$.

Notice that for example if $p=2$ then the trace theorem gives the bound $(1+\lambda)^{1 / 2}$ instead of $(1+\lambda)^{1 / 4}$ and thus Theorem 1 can be seen as an improvement of the trace theorem when the traces are taken from Laplace-Beltrami eigenfunctions.

Recall that the Weyl pointwise bound provides a constant $C$ such that

$$
\left\|\varphi_{\lambda}\right\|_{L^{\infty}(M)} \leq C(1+\lambda)^{\frac{1}{2}}\left\|\varphi_{\lambda}\right\|_{L^{2}(M)} .
$$

Therefore Theorem 11 may also be seen as an effect of the averaging along $\gamma$ of $\left|\varphi_{\lambda}\right|$ which may ${ }^{(1)}$ grow pointwisely much faster than $\lambda^{\frac{1}{4}}$ as $\lambda \rightarrow \infty$.

Let us denote by $\frac{D}{d t}$ the covariant derivative along $\gamma$ (recall that $\gamma$ is parametrized by arc length). For $2 \leq p<4$, estimate (1.1) is optimal for geodesic curves on the standard sphere $\mathbb{S}^{2}$, i.e. curves such that $\frac{D}{d t} \gamma^{\prime}=0$. The second goal of this paper is to show that estimate (1.1) in the range $2 \leq p<4$ can be significantly improved for curves with non vanishing geodesic curvature. We have the following statement.

Theorem 2. - Let $\gamma$ be such that

$$
g\left(\frac{D}{d t} \gamma^{\prime}, \frac{D}{d t} \gamma^{\prime}\right) \neq 0
$$

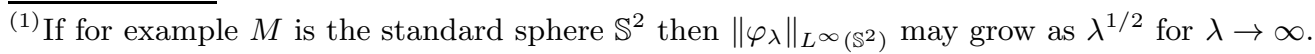


There exists a constant $C$ such that for every $\varphi_{\lambda}, 2 \leq p \leq 4$,

$$
\begin{gathered}
\left\|\varphi_{\lambda}\right\|_{L^{p}(\gamma)} \leq C(1+\lambda)^{\widetilde{\delta}(p)}\left\|\varphi_{\lambda}\right\|_{L^{2}(M)}, \\
\widetilde{\delta}(p)=\frac{1}{3}-\frac{1}{3 p}
\end{gathered}
$$

Moreover (1.4) is sharp in the case where $M$ is the standard sphere $\mathbb{S}^{2}$ and $\gamma$ is any curve with non vanishing geodesic curvature.

Remark 1.1. - In fact both theorems above still hold if one replace the eigenfunctions by the more general spectral $1_{|\sqrt{-\Delta}-\lambda| \leq 1}$. In that case, as will appear clearly in Section 5, the optimality holds for such spectral projector for any compact surface.

Let us remark that the techniques presented here extend to higher dimensions.

Theorem 3. - Let $(M, g)$ be a compact smooth Riemannian manifold of dimension $d$ and $\Sigma$ be a smooth submanifold of dimension $k$. There exists a constant $C>0$ such that for any $\varphi_{\lambda}$, we have

$$
\left\|\varphi_{\lambda}\right\|_{L^{p}(\Sigma)} \leq C(1+\lambda)^{\rho(k, d)}\left\|\varphi_{\lambda}\right\|_{L^{2}(M)}
$$

where

$$
\begin{aligned}
\rho(d-1, d) & = \begin{cases}\frac{d-1}{2}-\frac{d-1}{p} & \text { if } p_{0}=\frac{2 d}{d-1}<p \leq+\infty \\
\frac{d-1}{4}-\frac{d-2}{2 p} & \text { if } 2 \leq p<p_{0}=\frac{2 d}{d-1}\end{cases} \\
\rho(d-2, d) & =\frac{d-1}{2}-\frac{d-2}{p} \text { if } 2<p \leq+\infty \\
\rho(k, d) & =\frac{d-1}{2}-\frac{k}{p} \text { if } 1 \leq k \leq d-3
\end{aligned}
$$

If $p=p_{0}=\frac{2 d}{d-1}$ and $k=d-1$, we have

$$
\left\|\varphi_{\lambda}\right\|_{L^{p}(\Sigma)} \leq C(1+\lambda)^{\frac{d-1}{2 d}} \log ^{1 / 2}(\lambda)\left\|\varphi_{\lambda}\right\|_{L^{2}(M)}
$$

and if $p=2$ and $k=d-2$, we have

$$
\left\|\varphi_{\lambda}\right\|_{L^{p}(\Sigma)} \leq C(1+\lambda)^{\frac{1}{2}} \log ^{1 / 2}(\lambda)\left\|\varphi_{\lambda}\right\|_{L^{2}(M)}
$$

Moreover, all estimates are sharp, except for the log loss if

1. $k \leq d-2, M$ is the standard sphere $\mathbb{S}^{d}$ and $\Sigma$ is any submanifold of dimension $k$.

2. $k=d-1$ and $\frac{2 d}{d-1} \leq p \leq+\infty, M$ is the standard sphere $\mathbb{S}^{d}$ and $\Sigma$ is any hypersurface. 
3. $k=d-1$ and $2 \leq p<\frac{2 d}{d-1}, M$ is the standard sphere $M=\mathbb{S}^{d}$ and $\Sigma$ is any hypersurface containing a piece of geodesic.

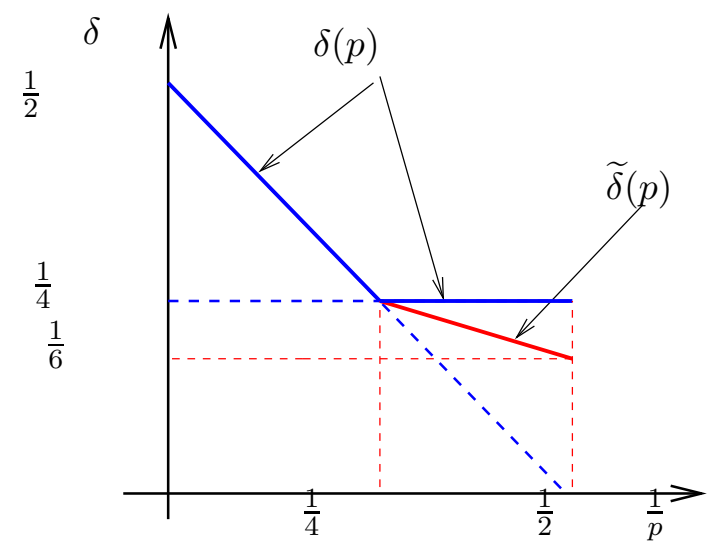

Figure 1. Curves on surfaces

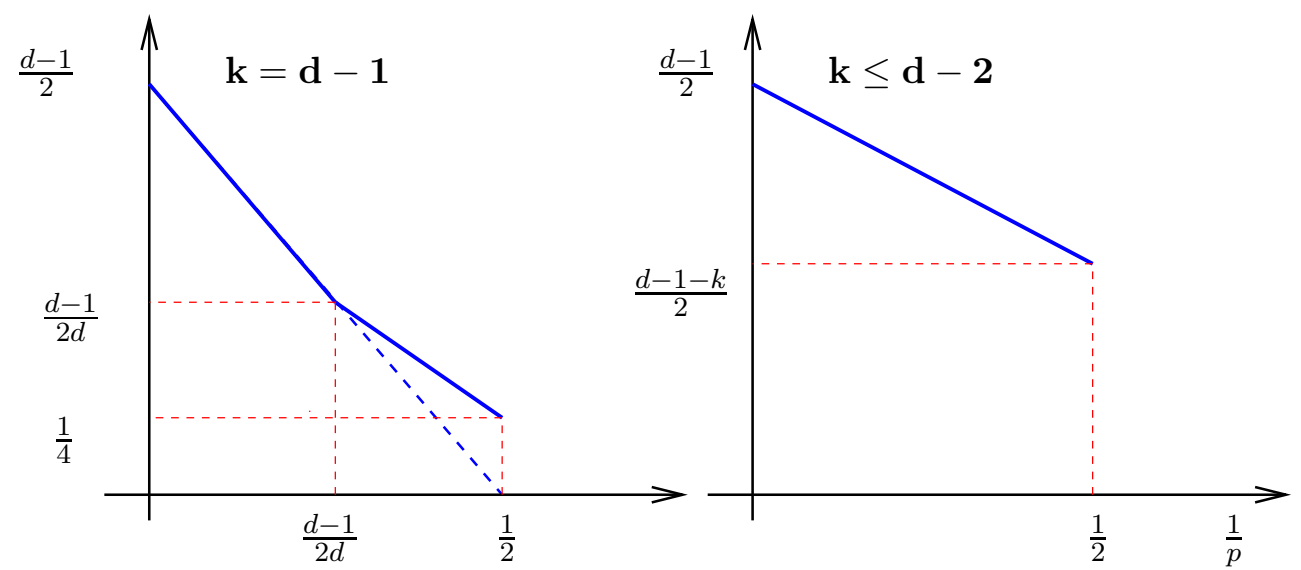

FiguRE 2. k-submanifolds in a d-manifold

When $(M, g)$ has negative constant curvature and in the special case $p=2$, estimate (1.1) when $\gamma$ is a geodesic and estimate (1.4) for $\gamma$ a geodesic circle were recently obtained by Reznikov [15. This work was a starting point and a motivation for our present work. It was only later that we realized that in fact our results in the special case of a hypersurface and $p=2$ can be deduced from Theorem 1 by Tataru [23] (considering an eigenfunction as a solution of the wave equation with a 
trivial time dependence). Remark that in turn Tataru's result is obtained as a consequence of classical estimates on Fourier integral operators with folded canonical relations by Melrose and Taylor 24 for the analog of our Theorem 2, (which in turn can be obtained from normal form results by Melrose 14, see also Hörmander, 12 , Theorem 25.3.11]) and by Greenleaf and Seeger [8] for the analog of our Theorem 1. To keep our paper essentially self contained, avoid the reader to go into the normal form machinery and maintain the technical nature of the exposition at a (rather) basic level, we elected to give a complete proof (including the case $p=2$ ).

It should be pointed out that estimates (1.1) and (1.4) are far from being optimal in the case of the flat torus $\mathbb{T}^{2}=\mathbb{R}^{2} /(2 \pi \mathbb{Z})^{2}$. In this case we have a strong improvement of the Weyl bound on the $L^{\infty}$ norm of the eigenfunctions. More precisely for every $\varepsilon>0$ there exists a constant $C_{\varepsilon}$ such that for every $\varphi_{\lambda}$,

$$
\left\|\varphi_{\lambda}\right\|_{L^{\infty}\left(\mathbb{T}^{2}\right)} \leq C_{\varepsilon}(1+\lambda)^{\varepsilon}\left\|\varphi_{\lambda}\right\|_{L^{2}\left(\mathbb{T}^{2}\right)} .
$$

Indeed, in the case of the flat torus we have the explicit representation of the eigenfunctions,

$$
\varphi_{\lambda}(x, y)=\sum_{m^{2}+n^{2}=\lambda^{2}} c_{m, n} e^{i(m x+n y)}, \quad c_{m, n} \in \mathbb{C} .
$$

Therefore (1.10) results from the Plancherel identity

$$
\left\|\varphi_{\lambda}\right\|_{L^{2}\left(\mathbb{T}^{2}\right)}^{2}=\sum_{m^{2}+n^{2}=\lambda^{2}}\left|c_{m, n}\right|^{2}
$$

the Cauchy-Schwarz inequality and the divisor bound

$$
\#\left((m, n) \in \mathbb{Z}^{2}: m^{2}+n^{2}=\lambda^{2}\right) \leq C_{\varepsilon}(1+\lambda)^{\varepsilon}, \quad \varepsilon>0
$$

in the ring of the Gaussian integers. Estimate (1.10) thus implies that for every curve $\gamma$ on $\mathbb{T}^{2}$, we have the bound

$$
\int_{\gamma}\left|\varphi_{\lambda}\right|^{2} \leq C_{\varepsilon}(1+\lambda)^{\varepsilon}\left\|\varphi_{\lambda}\right\|_{L^{2}\left(\mathbb{T}^{2}\right)}^{2}
$$

It would be interesting to decide whether one may replace $C_{\varepsilon}(1+\lambda)^{\varepsilon}$ in (1.11) by a constant uniform with respect to $\lambda$.

Let us mention that estimate (1.10) (and thus (1.11) is conjectured (see [16, 15]) to hold if the flat torus $\mathbb{T}^{2}$ is replaced by an arbitrary negatively curved manifold.

The paper is organized as follows. In Section 2 we recall the parametrix for a smoothed spectral projector (as written in 19] for example). Then we use this representation and a classical $T T^{\star}$ argument to prove (1.1) in the Section 3 In the Section 4 we precise the form of the parametrix and deduce (1.4) by analyzing precisely the oscillations which appear in the phase. In the Section 5, this precise description is used to prove the optimality of (1.1) and (1.4) on the sphere. In the 
last section we show how the two dimensional techniques can be adapted to prove Theorem 3 ,

Acknowledgements. A previous version of this article was only dealing with curves on surfaces. We thank one referee for pointing to us the question of higher dimension submanifolds and another one for various comments which improved the presentation of the paper and for proposing a counter example in section 5. We

also thank Melissa Tacy for pointing out an incorrect point in a previous proof of Lemma 5.3

\section{Representation of $\varphi_{\lambda}$ in local coordinates}

We have the following representation of $\varphi_{\lambda}$ in local coordinates.

Theorem 4. - There exits a function $\chi \in \mathcal{S}(\mathbb{R})$ such that $\chi(0)=1$ and for any $x_{0} \in M$ there exists systems of coordinates near $x_{0}$,

$$
W \subset V=\left\{x \in \mathbb{R}^{d}: x \sim 0\right\},
$$

a smooth function

$$
a: W_{x} \times V_{y} \times \mathbb{R}_{\lambda}^{+} \longrightarrow \mathbb{C}
$$

supported in the set

$$
\left\{(x, y) \in W \times V:|x| \leq c_{0} \varepsilon \leq c_{1} \varepsilon \leq|y| \leq c_{2} \varepsilon \ll 1\right\}
$$

and such that

$$
\forall \alpha \in \mathbb{N}^{2 d}, \exists C>0 ; \forall \lambda \geq 0,\left|\partial_{x, y} a(x, y, \lambda)\right| \leq C
$$

and an operator

$$
\mathcal{R}_{\lambda}: L^{2}(W) \longrightarrow L^{2}(W)
$$

such that,

$$
\left\|\mathcal{R}_{\lambda} f\right\|_{L^{\infty}(W)} \leq C\|f\|_{L^{2}(W)}
$$

and with $U:=W \cap\{x:|x| \leq c \varepsilon\}$, for every $x \in U$,

$$
\chi_{\lambda}(f):=\chi(\sqrt{-\boldsymbol{\Delta}}-\lambda) f=\lambda^{\frac{d-1}{2}} \int_{y \in V} e^{i \lambda \psi(x, y)} a(x, y, \lambda) f(y) d y+\mathcal{R}_{\lambda}(f),
$$

where $\psi(x, y)=-d_{g}(x, y)$ is the geodesic distance with respect to $g$ between $x$ and $y$. Furthermore, the symbol $a(x, y, \lambda)$ is real non negative and does not vanish for $|x| \leq c \varepsilon$ and $d_{g}(x, y) \in\left[c_{3} \varepsilon, c_{4} \varepsilon\right]$. 
Remark that since $\chi(\sqrt{-\boldsymbol{\Delta}}-\lambda) \varphi_{\lambda}=\varphi_{\lambda}$, relations (1.1) and (1.4) are consequences of similar estimates on the norm of the operator $\chi(\sqrt{-\boldsymbol{\Delta}}-\lambda)$ from $L^{2}(M)$ to $L^{p}(\gamma)$. Remark also that, since $\mathcal{R}_{\lambda}$ satisfies better estimates than we claimed in Theorems 1 and 2 , we will restrict the study to the analysis of the principal part in (2.1), $\mathcal{T}_{\lambda}$

$$
\mathcal{T}_{\lambda}(f)(x):=\int_{y \in V} e^{i \lambda \psi(x, y)} a(x, y) f(y) d y .
$$

The proof of Theorem 4 may be found in 19. Chap. 5] (except for the non vanishing of the symbol which comes from the explicit calculations made there) and is based on a representation of $\varphi_{\lambda}$ by the aid of the propagator of the wave equation

$$
\left(\partial_{t}^{2}-\Delta\right) u=0 \text {. }
$$

More precisely if $\chi \in \mathcal{S}(\mathbb{R}), \chi(0)=1$ is a Schwartz function such that $\operatorname{supp}(\widehat{\chi}) \subset$ $[\varepsilon, 2 \varepsilon]$ where $\varepsilon \ll 1$, then we can write

$$
\chi(\sqrt{-\boldsymbol{\Delta}}-\lambda) f=\frac{1}{2 \pi} \int_{\varepsilon}^{2 \varepsilon}\left(e^{i \tau \sqrt{-\boldsymbol{\Delta}}} f\right) e^{-i \tau \lambda} \widehat{\chi}(\tau) d \tau .
$$

For $\varepsilon \ll 1$ and $|\tau| \leq 2 \varepsilon$, introducing local coordinates near $x_{0} \in M$, we can represent $e^{i \tau \sqrt{-\Delta}}$ as a Fourier integral operator (see e.g. 10]). A stationary phase argument (see 19, Chap. 5]) in this representation then achieves the proof of Theorem 4.

\section{Proof of (1.1)}

In this section we give the proof of (1.1). Let $x_{0} \in \gamma$. Using a partition of unity, we may assume that $\gamma$ is contained in the domain $U$ of our coordinate patch defined in Theorem 4 and $x_{0}=0$. We assume that $\gamma$ is parametrized by arc length, $s$ and define

$$
T_{\lambda}(f)(s)=\mathcal{T}_{\lambda}(f)(x(s)) .
$$

Therefore it suffices to prove that

$$
\left\|T_{\lambda} f\right\|_{L^{p}(\gamma)} \leq C \lambda^{\delta(p)-\frac{1}{2}}\left(\int_{y \in V}|f(y)|^{2} d y\right)^{1 / 2} .
$$

We represent $y$ in geodesic polar coordinates as $y=\exp _{0}(r \omega), r>0, \omega \in \mathbb{S}^{1}$ and $c_{1} \varepsilon \leq r \leq c_{2} \varepsilon$. We can write

$$
\left(T_{\lambda} f\right)(x)=\int_{c_{1} \varepsilon}^{c_{2} \varepsilon}\left(T_{\lambda}^{r} f_{r}\right)(x) d r
$$

where

$$
\left(T_{\lambda}^{r} f\right)(x)=\int_{\mathbb{S}^{1}} e^{i \lambda \psi_{r}(x, \omega)} a_{r}(x, \omega) f(\omega) d \omega
$$


with

$$
\psi_{r}(x, \omega)=\psi(x, y), \quad f_{r}(\omega)=f(y), \quad a_{r}(x, \omega)=\kappa(r, \omega) a(x, y)
$$

for some smooth function $\kappa$. It suffices consequently to show the bound

$$
\left\|T_{\lambda}^{r} f\right\|_{L^{p}(\gamma)} \leq C \lambda^{\delta(p)-\frac{1}{2}}\left(\int_{\mathbb{S}^{1}}|f(\omega)|^{2} d \omega\right)^{1 / 2} .
$$

Indeed if we suppose that (3.2) is true, then we can write

$$
\begin{aligned}
& \left\|T_{\lambda} f\right\|_{L^{p}(\gamma)} \leq \int_{c_{1} \varepsilon}^{c_{2} \varepsilon}\left\|T_{\lambda}^{r} f_{r}\right\|_{L^{p}(\gamma)} d r \\
& \quad \leq C \lambda^{\delta(p)-\frac{1}{2}} \int_{c_{1} \varepsilon}^{c_{2} \varepsilon}\left\|f_{r}\right\|_{L^{2}\left(\mathbb{S}^{1}\right)} d r \leq c \lambda^{\delta(p)-\frac{1}{2}}\|f\|_{L^{2}(V)}
\end{aligned}
$$

which is (3.1). Thus it remains to prove (3.2). For that purpose we use a duality argument. Computing $\left\|\left(T_{\lambda}^{r}\right)^{\star} f\right\|_{L^{2}}^{2}$, we can apply the usual $T-T^{\star}$ argument:

$$
\begin{aligned}
\left\|T_{\lambda}^{r}\right\|_{\mathcal{L}\left(L^{2}\left(\mathbb{S}^{1}\right) ; L^{p}(\gamma)\right)}^{2} & =\left\|\left(T_{\lambda}^{r}\right)^{\star}\right\|_{\mathcal{L}\left(L^{p^{\prime}}(\gamma) ; L^{2}\left(\mathbb{S}^{1}\right)\right)}^{2} \\
& =\left\|T_{\lambda}^{r}\left(T_{\lambda}^{r}\right)^{\star}\right\|_{\mathcal{L}\left(L^{p^{\prime}}(\gamma) ; L^{p}(\gamma)\right)}
\end{aligned}
$$

A direct computation shows that

$$
\left(T_{\lambda}^{r}\left(T_{\lambda}^{r}\right)^{\star} f\right)(x(t))=\int_{a}^{b} K(t, \tau) f(x(\tau)) d \tau
$$

where

$$
K(t, \tau)=\int_{\mathbb{S}^{1}} e^{i \lambda\left[\psi_{r}(x(t), \omega)-\psi_{r}(x(\tau), \omega)\right]} a_{r}(x(t), \omega) \overline{a_{r}}(x(\tau), \omega) d \omega .
$$

One can calculate the traces at zero of the first order derivatives of the map

$$
x \longrightarrow \psi_{r}(x, \omega) \text {. }
$$

More precisely, we have the following statement.

Lemma 3.1. - Suppose that $g(0)=\mathrm{Id}$. Then for $\varepsilon \ll 1$,

$$
\left(\partial_{x_{1}} \psi_{r}(0, \omega), \partial_{x_{2}} \psi_{r}(0, \omega)\right)=\omega, \quad \omega=\left(\omega_{1}, \omega_{2}\right) \in \mathbb{S}^{1} .
$$

Proof. - The proof of Lemma 3.1 may be found in 3]. For the sake of completeness, we recall it below. Let $y=\exp _{0}(r \omega)$ and $u=u(x, y) \in T_{y} M$ be the unit vector such that

$$
\exp _{y}(-\psi(x, y) u(x, y))=x .
$$

Differentiating with respect to $x$ this identity, we get for $x=0$, and any $h \in T_{0} M$,

$$
h=T_{r u(0, y)}\left(\exp _{y}\right)\left[-d_{x} \psi(0, y) \cdot h u(0, y)-r T_{x} u(0, y) \cdot h\right] \text {. }
$$


But since $y=\exp _{0}(r \omega)$

$$
T_{r u(0, y)}\left(\exp _{y}\right) \cdot u(0, y)=-\omega
$$

Take the scalar product with $\omega$ in (3.5). Using Gauss' Lemma (see for example 6 , $3.70]$ ), we get

$$
d_{x} \psi(0, y) \cdot h=g_{0}(h, \omega)
$$

i.e.

$$
\nabla_{x} \psi(0, y)=\omega
$$

Note that by performing a linear change of coordinates, we can assume that $g(0)=$ Id. Using Lemma 3.1. we can estimate the kernel $K(t, \tau)$.

Lemma 3.2. - There exists $\delta>0$ such that for $|t-\tau|<\delta$,

$$
|K(t, \tau)| \lesssim(1+\lambda|t-\tau|)^{-\frac{1}{2}} .
$$

Proof. - For $\left(x, x^{\prime}\right) \in U \times U$, we set

$$
K\left(x, x^{\prime}\right)=\int_{\mathbb{S}^{1}} e^{i \lambda\left[\psi_{r}(x, \omega)-\psi_{r}\left(x^{\prime}, \omega\right)\right]} a_{r}(x, \omega) \overline{a_{r}}\left(x^{\prime}, \omega\right) d \omega .
$$

We are going to show that for $x, x^{\prime}$ close to zero,

$$
\left|K\left(x, x^{\prime}\right)\right| \lesssim\left(1+\lambda\left|x-x^{\prime}\right|\right)^{-\frac{1}{2}}
$$

which, in view of the property $g(\dot{x}(t), \dot{x}(t))=1$, implies the assertion of the lemma. Let us now give the proof of (3.7). We parametrize the circle $\mathbb{S}^{1}$ as

$$
\omega=\omega(w)=(\cos w, \sin w), \quad w \in[0,2 \pi] .
$$

The Taylor formula allows to write

$$
\psi_{r}(x, \omega)-\psi_{r}\left(x^{\prime}, \omega\right)=\left\langle x-x^{\prime}, \Psi\left(x, x^{\prime}, \omega\right)\right\rangle,
$$

where

$$
\Psi\left(x, x^{\prime}, \omega\right)=\int_{0}^{1} \nabla_{x} \psi_{r}\left(x^{\prime}+\theta\left(x-x^{\prime}\right), \omega\right) d \theta .
$$

In (3.8), (3.9), $\langle\cdot, \cdot\rangle$ stands for the $\mathbb{R}^{2}$ scalar product and $\nabla_{x}=\left(\partial_{x_{1}}, \partial_{x_{2}}\right)$. We can therefore write

$$
\psi_{r}(x, \omega)-\psi_{r}\left(x^{\prime}, \omega\right)=\left|x-x^{\prime}\right| \Phi\left(x, x^{\prime}, \sigma, \omega\right),
$$

where

$$
\Phi\left(x, x^{\prime}, \sigma, \omega\right)=\left\langle\sigma, \Psi\left(x, x^{\prime}, \omega\right)\right\rangle, \quad \sigma=\frac{x-x^{\prime}}{\left|x-x^{\prime}\right|} \in \mathbb{S}^{1}
$$


For $\left(x, x^{\prime}, \sigma\right) \in U \times U \times \mathbb{S}^{1}$ we set

$$
K_{1}\left(x, x^{\prime}, \sigma\right):=\int_{\mathbb{S}^{1}} e^{i \lambda \Phi\left(x, x^{\prime}, \sigma, \omega\right)} a_{r}(x, \omega) \overline{a_{r}}\left(x^{\prime}, \omega\right) d \omega .
$$

It suffices therefore to show that there exist $\varepsilon \ll 1$ and $C>0$ such that for every $\left(x, x^{\prime}, \sigma\right) \in U \times U \times \mathbb{S}^{1}$, every $\lambda \geq 0$,

$$
\left|K_{1}\left(x, x^{\prime}, \sigma\right)\right| \leq C(1+\lambda)^{-\frac{1}{2}} .
$$

Suppose that

$$
\sigma=(\cos \alpha, \sin \alpha), \quad \alpha \in[0,2 \pi] .
$$

Thanks to Lemma 3.1 .

$$
\Phi(0,0, \sigma, \omega(w))=\langle\sigma, \omega\rangle=\cos (w-\alpha) .
$$

Next, we can write

$$
\Phi_{w}^{\prime}(0,0, \sigma, \omega(w))=-\sin (w-\alpha)
$$

and

$$
\Phi_{w w}^{\prime \prime}(0,0, \sigma, \omega(w))=-\cos (w-\alpha) .
$$

The main point is that $\Phi_{w}^{\prime}(0,0, \sigma, \omega(w))$ and $\Phi_{w w}^{\prime \prime}(0,0, \sigma, \omega(w))$ cannot vanish simultaneously. In view of (3.10) and (3.11) we can represent the circle $\mathbb{S}^{1}$ as a disjoint union of 4 segments $I_{1}(\sigma), I_{2}(\sigma), I_{3}(\sigma)$ and $I_{4}(\sigma)$, where $I_{2}(\sigma)$ and $I_{4}(\sigma)$ are neighborhoods of $\alpha$ and $\alpha+\pi$ respectively, so that

$$
\left|\Phi_{w}^{\prime}(0,0, \sigma, \omega(w))\right| \geq \frac{\sqrt{2}}{2}, \quad w \in I_{1}(\sigma) \cup I_{3}(\sigma)
$$

and

$$
\left|\Phi_{w w}^{\prime \prime}(0,0, \sigma, \omega(w))\right| \geq \frac{\sqrt{2}}{2}, \quad w \in I_{2}(\sigma) \cup I_{4}(\sigma) .
$$

Therefore, by continuity, there exists $\varepsilon \ll 1$ such that for every $\sigma \in \mathbb{S}^{1}$, if

$$
\left(x, x^{\prime}\right) \in U \times U
$$

then

$$
\left|\Phi_{w}^{\prime}\left(x, x^{\prime}, \sigma, \omega(w)\right)\right| \geq \frac{\sqrt{2}}{4}, \quad w \in I_{1}(\sigma) \cup I_{3}(\sigma)
$$

and

$$
\left|\Phi_{w w}^{\prime \prime}\left(x, x^{\prime}, \sigma, \omega(w)\right)\right| \geq \frac{\sqrt{2}}{4}, \quad w \in I_{2}(\sigma) \cup I_{4}(\sigma) .
$$


We now split the integral defining $K_{1}\left(x, x^{\prime}, \sigma\right)$ in 4 parts according to the splitting of $\mathbb{S}^{1}$ to $I_{1}(\sigma), I_{2}(\sigma), I_{3}(\sigma)$ and $I_{4}(\sigma)$. Thanks to (3.12) and an integration by parts the contributions of $I_{1}(\sigma)$ and $I_{3}(\sigma)$ are bounded by

$$
C(1+\lambda)^{-1} \text {. }
$$

Thanks to (3.13) and the basic van der Corput lemma (see 21, Chap VIII.1]) the contributions of $I_{2}(\sigma)$ and $I_{4}(\sigma)$ are bounded by

$$
C(1+\lambda)^{-\frac{1}{2}}
$$

This completes the proof of Lemma 3.2

Let us now complete the proof of Theorem 11. We first recall the following classical consequence of Young's and Hardy-Littlewood's inequalities

Lemma 3.3. - Consider $2 \leq p \leq+\infty$ and $T$ a convolution operator

$$
T f(x)=\int_{-\infty}^{+\infty} K(x-y) f(y) d y
$$

with $K \in L_{\text {loc. }}^{1}$. Then the norm of $T$ as an operator from $L^{p^{\prime}}(\mathbb{R})$ to $L^{p}(\mathbb{R})$ is bounded by $\|K\|_{L^{\frac{p}{2}}}$. Furthermore, if $K(x)=\frac{1}{|x|^{2 / p}}$ and $p \neq 2$, then the operator $T$ is still bounded from $L^{p^{\prime}}(\mathbb{R})$ to $L^{p}(\mathbb{R})$ (despite the logarithmic divergence of $\|K\|_{L^{\frac{p}{2}(\mathbb{R})}}$ ).

Using Lemma 3.2 we can write

$$
\begin{aligned}
\left\|T_{\lambda}^{r}\left(T_{\lambda}^{r}\right)^{\star} f\right\|_{L^{p}(\gamma)} & \lesssim\left\|\int_{a}^{b}(1+\lambda|t-\tau|)^{-\frac{1}{2}}|f(x(\tau))| d \tau\right\|_{L^{p}(a, b)} \\
& \lesssim\left\|(1+\lambda|t|)^{-\frac{1}{2}}\right\|_{L^{\frac{p}{2}(a, b)}}\|f\|_{L^{p^{\prime}}(\gamma)} .
\end{aligned}
$$

But

$$
\left\|(1+\lambda|t|)^{-\frac{1}{2}}\right\|_{L^{\frac{p}{2}(a, b)}} \leq \lambda^{-\frac{2}{p}}\left(\int_{0}^{C \lambda}(1+\tau)^{-\frac{p}{4}} d \tau\right)^{\frac{2}{p}} \lesssim \begin{cases}\lambda^{-\frac{1}{2}} & \text { if } 2 \leq p<4 \\ \lambda^{-\frac{2}{p}} & \text { if } 4<p\end{cases}
$$

Therefore for $p \neq 4 T_{\lambda}^{r}\left(T_{\lambda}^{r}\right)^{\star}$ sends $L^{p^{\prime}}(\gamma)$ to $L^{p}(\gamma)$ with operator norm $\leq C \lambda^{\delta(p)-\frac{1}{2}}$. The case $p=4$ follows from the additional property in Lemma 3.3. This ends the proof of Theorem 1 (except for the optimality which will be dealt with in section 5). 


\section{Proof of (1.4)}

This section is devoted to the proof of (1.4). For that purpose we need a more precise description of the kernel of the operators which appeared in the previous section. Notice that this section contains all the results in section 3, but we preferred to keep the technical level of the exposition as low as possible for the proof of Theorem 1. To prove (1.4) we only need to consider the case $p=2$ (all the other cases being deduced from that one and the special case $p=4$ in Theorem 1 by interpolation).

As in the proof of (1.1), for $x_{0} \in \gamma$ and $\lambda \geq 1$, we consider the map

$$
\left(T_{\lambda} f\right)(x):=\int_{y \in V} e^{i \lambda \psi(x, y)} a(x, y) f(y) d y, \quad x \in U,
$$

where $U$ and $V$ are coordinate systems near $x_{0}$ given in Theorem [4 $a(x, y)$ is the amplitude introduced in the statement of Theorem 4 and $-\psi(x, y)$ is the geodesic distance between $x$ and $y$. Estimate (1.4) is therefore a consequence of the estimate

$$
\left\|T_{\lambda} f\right\|_{L^{2}(\gamma)}^{2} \leq C \lambda^{-\frac{2}{3}} \int_{y \in V}|f(y)|^{2} d y .
$$

Recall that the $L^{2}(\gamma)$ norm measures the contribution of $U$ to the $L^{2}$ norm along $\gamma$. By duality it suffices to show that $T_{\lambda}^{\star}$ sends $L^{2}(V)$ to $L^{2}(\gamma)$ with norm $\leq C \lambda^{-\frac{1}{3}}$. Finally, both previous statements are equivalent to the continuity of $T_{\lambda} T_{\lambda}^{\star}$ from $L^{2}(\gamma)$ to $L^{2}(\gamma)$ with operator norm $\leq C \lambda^{-\frac{2}{3}}$. As in the proof of (1.1) the main point in the proof of (1.4) is a precise description of the kernel of $T_{\lambda} T_{\lambda}^{\star}$. The new fact is that an oscillatory factor in the kernel of $T_{\lambda} T_{\lambda}^{\star}$ will be crucial to achieve the needed bound. Recall that in the proof of (1.1) only pointwise bounds on the kernel of $T_{\lambda} T_{\lambda}^{\star}$ were used. We can write

$$
\left(T_{\lambda} T_{\lambda}^{\star}\right)(f)(x(t))=\int_{a}^{b} K(t, \tau) f(x(\tau)) d \tau,
$$

where

$$
K(t, \tau)=\mathcal{K}(x(t), x(\tau))
$$

with

$$
\mathcal{K}\left(x, x^{\prime}\right)=\int_{V} e^{i \lambda\left[\psi(x, y)-\psi\left(x^{\prime}, y\right)\right]} a(x, y) \bar{a}\left(x^{\prime}, y\right) d y .
$$

We now give a precise description of this kernel. 
Lemma 4.1. - There exist $\varepsilon \ll 1, a^{ \pm}, b \in C^{\infty}\left(\mathbb{R}^{2} \times \mathbb{R}^{2} \times \mathbb{R}\right)$ such that for $\left|x-x^{\prime}\right| \gtrsim$ $\lambda^{-1}, x, x^{\prime} \in U$,

$$
\mathcal{K}\left(x, x^{\prime}\right)=\sum_{ \pm} \frac{e^{ \pm i \lambda \psi\left(x, x^{\prime}\right)}}{\left(\lambda\left|x-x^{\prime}\right|\right)^{\frac{1}{2}}} a^{ \pm}\left(x^{\prime}, x-x^{\prime}\right)+b\left(x, x^{\prime}, \lambda^{-1}\left|x-x^{\prime}\right|^{-1}\right) .
$$

Moreover $a^{ \pm}$are real, have supports of size $\mathcal{O}(\varepsilon)$ with respect to the $x$ and $x^{\prime}$ variables and $a^{ \pm}(0,0) \geq c>0$. Finally

$$
\left|b\left(x, x^{\prime}, \lambda^{-1}\left|x-x^{\prime}\right|^{-1}\right)\right| \leq C\left(\lambda\left|x-x^{\prime}\right|\right)^{-\frac{3}{2}} .
$$

Remark 4.2. - We have $\chi_{\lambda} \chi_{\lambda}^{\star}=\left(|\chi|^{2}\right)_{\lambda}$. As a consequence, the lemma above amounts to describing the kernel of $|\chi|_{\lambda}^{2}$. The main difference with respect to Sogge's result in Theorem 4 is that the Fourier transform of $|\chi|^{2}$ no longer vanishes near $t=0$ which makes the description more difficult.

Proof. - We represent $y$ in geodesic polar coordinates with origin at $x^{\prime}$ as

$$
y=\exp _{x^{\prime}}(r \omega), \quad r>0, \quad \omega \in \mathbb{S}^{1} .
$$

Denote by

$$
b_{r}\left(x, x^{\prime}, \omega\right)=a\left(x, \exp _{x^{\prime}}(r \omega)\right) \bar{a}\left(x^{\prime}, \exp _{x^{\prime}}(r \omega)\right) \kappa\left(x^{\prime}, r, \omega\right) .
$$

We can therefore write

$$
\mathcal{K}\left(x, x^{\prime}\right)=\int_{r=c_{1} \varepsilon}^{c_{2} \varepsilon} \int_{\mathbb{S}^{1}} e^{i \lambda\left[\psi_{r}(x, \omega)-\psi_{r}\left(x^{\prime}, \omega\right)\right]} b_{r}\left(x, x^{\prime}, \omega\right) d \omega d r,
$$

where $\kappa(r)$ is a smooth positive function and

$$
\psi_{r}(x, \omega)=\psi(x, y)=-d_{g}\left(x, \exp _{x^{\prime}}(r \omega)\right) .
$$

We are going to evaluate the integral over $\omega$ in (4.1) by the stationary phase formula. Assume that

$$
\omega=(\cos w, \sin w), \quad w \in[0,2 \pi] .
$$

We are going to show that for $x \neq x^{\prime}$, close to each other, the map

$$
\omega \longrightarrow \psi_{r}(x, \omega)-\psi_{r}\left(x^{\prime}, \omega\right)
$$

from $\mathbb{S}^{1}$ to $\mathbb{R}$ has exactly two non degenerate critical points. By the fundamental theorem of calculus

$$
\psi_{r}(x, \omega)-\psi_{r}\left(x^{\prime}, \omega\right)=\left|x-x^{\prime}\right| \int_{0}^{1}\left\langle\sigma, \nabla_{x} \psi_{r}\left(x^{\prime}+\theta\left(x-x^{\prime}\right), \omega\right)\right\rangle d \theta, \quad \sigma=\frac{x-x^{\prime}}{\left|x-x^{\prime}\right|} .
$$

Further, we consider the real valued function $\Phi_{r}$ on $\mathbb{S}^{1} \times U \times U \times \mathbb{S}^{1}$, defined by

$$
\Phi_{r}\left(\sigma, x, x^{\prime}, \omega\right):=\int_{0}^{1}\left\langle\sigma, \nabla_{x} \psi_{r}\left(x^{\prime}+\theta\left(x-x^{\prime}\right), \omega\right)\right\rangle d \theta .
$$


Let us show that for fixed $\sigma \in \mathbb{S}^{1}$ and $x, x^{\prime}$ close to each other, the map

$$
w \longrightarrow \Phi_{r}\left(\sigma, x, x^{\prime}, \omega(w)\right)
$$

from $[0,2 \pi]$ to $\mathbb{R}$ has exactly two non degenerate critical points. We can assume that $g\left(x^{\prime}\right)=$ Id and using Lemma 3.1 we obtain

$$
\Phi_{r}\left(\sigma, x^{\prime}, x^{\prime}, \omega\right)=\langle\sigma, \omega\rangle
$$

and therefore

$$
\frac{\partial \Phi_{r}}{\partial w}\left(\sigma, x^{\prime}, x^{\prime}, \omega(w)\right)=\langle\sigma, \dot{\omega}\rangle,
$$

where $\dot{\omega}=(-\sin w, \cos w)$ denotes the first derivative of $\omega$ with respect to $w$. Hence, for fixed $\sigma \in \mathbb{S}^{1}$ the equation

$$
\frac{\partial \Phi_{r}}{\partial w}\left(\sigma, x^{\prime}, x^{\prime}, \omega\right)=0
$$

has exactly two solutions $\omega= \pm \sigma$. Clearly

$$
\frac{\partial^{2} \Phi_{r}}{\partial w^{2}}\left(\sigma, x^{\prime}, x^{\prime}, \omega\right)=\langle\sigma, \ddot{\omega}\rangle,
$$

where $\ddot{\omega}=(-\cos w,-\sin w)$ denotes the second derivative of $\omega$ with respect to $w$. Therefore

$$
\left|\frac{\partial^{2} \Phi_{r}}{\partial w^{2}}\left(\sigma, x^{\prime}, x^{\prime}, \pm \sigma\right)\right|=1 .
$$

Thanks to the implicit function theorem ${ }^{(2)}$, we obtain that there exist $\delta>0, \delta^{\prime}>0$ such that for every $\sigma \in \mathbb{S}^{1}$, every $\left|x-x^{\prime}\right|<\delta$ there exists a unique $\omega \in \mathbb{S}^{1}$ satisfying $|\omega-\sigma|<\delta^{\prime}$ which is a non degenerate critical point of (4.3). Moreover there exists a unique $\omega \in \mathbb{S}^{1}$ satisfying $|\omega+\sigma|<\delta^{\prime}$ which is a non degenerate critical point of (4.3). Moreover for $\omega$ outside the intervals $|\omega-\sigma|<\delta^{\prime}$ and $|\omega+\sigma|<\delta^{\prime}$, thanks to (4.4),

$$
\left|\frac{\partial \Phi_{r}}{\partial w}\left(\sigma, x, x^{\prime}, \omega\right)\right| \geq c>0
$$

provided $x, x^{\prime}$ being close to each other. We therefore obtain that (4.3) has exactly two non degenerate critical points. This in turn implies that for $x \neq x^{\prime}$ the map (4.2) has also exactly two non degenerate critical points.

Our next step is to construct explicitly the critical points of (4.2). Let us denote by $\Sigma$ the circle centered at $x^{\prime}$ and of radius $r$ in the geodesic coordinate system with center $x^{\prime}$ (see Figure 2). The geodesic $l$ joining the (different) points $x$ and $x^{\prime}$ is a straight line in this coordinate system. Let us denote by $\exp _{x^{\prime}}\left(r \omega^{\star}\right)$ and $\exp _{x^{\prime}}\left(r\left(\omega^{\star}+\pi\right)\right)$ the intersections of $l$ and $\Sigma$. We can clearly suppose that $x$ lies

${ }^{(2)}$ Notice the uniform dependence of $\delta, \delta^{\prime}$ with respect to $\sigma \in \mathbb{S}^{1}$. 
on the segment between $x^{\prime}$ and $\exp _{x^{\prime}}\left(r \omega^{\star}\right)$. We are now going to show that $\omega^{\star}$ and

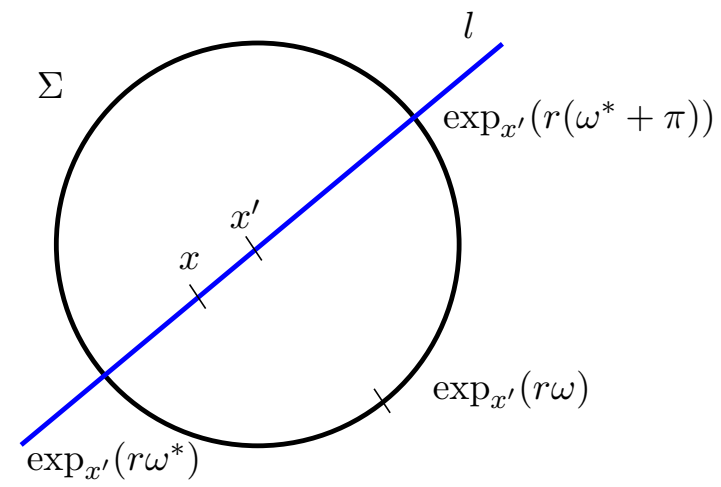

FiguRE 3 .

$\omega^{\star}+\pi$ are (the) critical points of (4.2). Let $\exp _{x^{\prime}}(r \omega), \omega \in \mathbb{S}^{1}$ be an arbitrary point on $\Sigma$. The triangle inequality yields

$$
d_{g}\left(x^{\prime}, \exp _{x^{\prime}}(r \omega)\right)-d_{g}\left(x, x^{\prime}\right) \leq d_{g}\left(x, \exp _{x^{\prime}}(r \omega)\right) \leq d_{g}\left(x, x^{\prime}\right)+d_{g}\left(x^{\prime}, \exp _{x^{\prime}}(r \omega)\right) .
$$

Notice that $d_{g}\left(x^{\prime}, \exp _{x^{\prime}}(r \omega)\right)=r$, and moreover using that the line joining $x$ and $x^{\prime}$ is a geodesic, we infer the identities

$$
d_{g}\left(x, x^{\prime}\right)+d_{g}\left(x, \exp _{x^{\prime}}\left(r \omega^{\star}\right)\right)=r, \quad d_{g}\left(x, \exp _{x^{\prime}}\left(r\left(\omega^{\star}+\pi\right)\right)\right)-d_{g}\left(x, x^{\prime}\right)=r .
$$

Therefore, in view of (4.5),

$$
d_{g}\left(x, \exp _{x^{\prime}}\left(r \omega^{\star}\right)\right) \leq d_{g}\left(x, \exp _{x^{\prime}}(r \omega)\right) \leq d_{g}\left(x, \exp _{x^{\prime}}\left(r\left(\omega^{\star}+\pi\right)\right)\right)
$$

and equalities are possible if and only if $\omega=\omega^{\star}$ or $\omega=\omega^{\star}+\pi$. But (4.6) can be rewritten as

$$
\psi_{r}\left(x, \omega^{\star}\right) \leq \psi_{r}(x, \omega) \leq \psi_{r}\left(x, \omega^{\star}+\pi\right), \quad \forall \omega \in \mathbb{S}^{1} .
$$

On the other hand

$$
\psi_{r}\left(x^{\prime}, \omega\right)=r, \quad \forall \omega \in \mathbb{S}^{1}
$$

and therefore $\omega^{\star}$ is the unique global minimum of (4.2) while $\omega^{\star}+\pi$ is the unique global maximum of (4.2). Hence, for $x \neq x^{\prime}$, the critical points of (4.2) are $\omega^{\star}$ and $\omega^{\star}+\pi$.

We next evaluate the value of $\psi_{r}(x, \omega)-\psi_{r}\left(x^{\prime}, \omega\right)$ at the critical points. Since the line joining $x$ and $x^{\prime}$ is a geodesic, we obtain that

$$
\psi_{r}\left(x, \omega^{\star}\right)-\psi_{r}\left(x^{\prime}, \omega^{\star}\right)=-d_{g}\left(x, x^{\prime}\right)=\psi\left(x, x^{\prime}\right) .
$$

Similarly,

$$
\psi_{r}\left(x, \omega^{\star}+\pi\right)-\psi_{r}\left(x^{\prime}, \omega^{\star}+\pi\right)=d_{g}\left(x, x^{\prime}\right)=-\psi\left(x, x^{\prime}\right) .
$$


Therefore the values of the phase $\psi_{r}(x, \omega)-\psi_{r}\left(x^{\prime}, \omega\right)$ in the critical points are $\pm \psi\left(x, x^{\prime}\right)$. Note that this value is independent of $r$. Coming back to (4.1) we apply the stationary phase formula to the integral over $\omega$ and since $\pm \psi\left(x, x^{\prime}\right)$ is independent of $r$, after the integration over $r$, we arrive at the claimed representation for the kernel $\mathcal{K}\left(x, x^{\prime}\right)$. The non vanishing of $a^{ \pm}(0,0)$ simply comes from the explicit representation we get from the stationary phase argument and the non negativeness of the function $a$ appearing in Theorem 4 This reads

$$
a^{ \pm}\left(0, x^{\prime}\right)=\int_{r} \Psi\left(x, x^{\prime}, r\right) b_{r}\left(x, x^{\prime}, \omega^{\star}+\left( \pm \frac{\pi}{2}-\frac{\pi}{2}\right)\right) d r
$$

where $\Psi\left(x, x^{\prime}, r\right)$ is a non vanishing function (the Jacobian determinant). This completes the proof of Lemma 4.1 .

Let $\chi \in C_{0}^{\infty}(\mathbb{R})$ such that

$$
\chi(x)= \begin{cases}1, & |x| \leq 1 / 2 \\ 0, & |x| \geq 1\end{cases}
$$

and $\zeta \in C_{0}^{\infty}(\mathbb{R})$ such that

$$
\zeta(x)= \begin{cases}1, & |x| \leq 1 / 8 \\ 0, & |x| \geq 1 / 4\end{cases}
$$

Then, since for $\lambda \geq 1$,

$$
(1-\zeta)(\lambda x)(1-\chi)\left(\lambda^{1 / 3} x\right)=(1-\chi)\left(\lambda^{1 / 3} x\right)
$$

we can write

$$
1=\zeta(\lambda(t-\tau))+(1-\zeta)(\lambda(t-\tau))(1-\chi)\left(\lambda^{1 / 3}(t-\tau)\right)+\chi\left(\lambda^{1 / 3}(t-\tau)\right) .
$$

According to the above partition of the unity, we split the kernel as

$$
K(t, \tau)=K_{1}(t, \tau)+K_{2}(t, \tau)+K_{3}(t, \tau)
$$

which in turn induces a natural splitting of $T_{\lambda} T_{\lambda}^{\star}$ as

$$
T_{\lambda} T_{\lambda}^{\star}=L_{1}+L_{2}+L_{3}
$$

where $L_{j}, j=1,2,3$ denote the contributions of $K_{j}$ to $T_{\lambda} T_{\lambda}^{\star}$ respectively. Using the Young inequality and a crude bound on $K(t, \tau)$, we obtain the estimate

$$
\left\|L_{1}(f)\right\|_{L^{2}(\gamma)} \leq C\|\zeta(\lambda t)\|_{L^{1}(\mathbb{R})}\|f\|_{L^{2}(\gamma)} \leq C \lambda^{-1}\|f\|_{L^{2}(\gamma)}
$$

which is even better than the needed estimate. Next we estimate $L_{2}$ whose kernel in supported in the set

$$
\left\{(t, \tau): \lambda^{-1} \lesssim|t-\tau| \lesssim \lambda^{-1 / 3}\right\}
$$


Using Lemma 4.1, we can write

$$
\left|K_{2}(t, \tau)\right| \leq \frac{C\left|(1-\zeta)(\lambda(t-\tau)) \chi\left(\lambda^{1 / 3}(t-\tau)\right)\right|}{(\lambda|t-\tau|)^{\frac{1}{2}}} .
$$

Using the Young inequality, we get

$$
\begin{aligned}
\left\|L_{2}(f)\right\|_{L^{2}(\gamma)} & \leq \frac{C}{\lambda^{1 / 3}}\left\|\frac{(1-\zeta)(\lambda t) \chi\left(\lambda^{1 / 3} t\right)}{\left(\lambda^{1 / 3}|t|\right)^{\frac{1}{2}}}\right\|_{L^{1}(\mathbb{R})}\|f\|_{L^{2}(\gamma)} \\
& \leq C \lambda^{-\frac{2}{3}}\|f\|_{L^{2}(\gamma)} .
\end{aligned}
$$

It remains to estimate $L_{3}$ whose kernel is supported in the set

$$
\left\{(t, \tau): \lambda^{-1 / 3} \lesssim|t-\tau|\right\} .
$$

For $t, \tau$ on the support of $(1-\chi)\left(\lambda^{1 / 3}(t-\tau)\right)$, with the notation of Lemma 4.1, we can write

$$
\left|b\left(x(t), x(\tau), \lambda^{-1}|x(t)-x(\tau)|^{-1}\right)\right| \leq C(\lambda|x(t)-x(\tau)|)^{-3 / 2} \leq C^{\prime} \lambda^{-1} .
$$

Therefore, the contribution of $b$ to $L_{3}$ is bounded by $\lambda^{-1}\|f\|_{L^{2}(\gamma)}$ and we only need to estimate the contribution of the first term in the representation of $\mathcal{K}\left(x, x^{\prime}\right)$ given by Lemma 4.1

Our next step is a general property of curves passing through the origin of a geodesic coordinate system.

Lemma 4.3. - Let

$$
x(t)=\left(x_{1}(t), x_{2}(t)\right), t \sim 0
$$

be a smooth curve in geodesic coordinate system with origin 0 , parametrized by its arc length. Suppose also that $x(0)=0$ and $g(0)=\mathrm{Id}$. Then

$$
|\dot{x}(0)|=1, \quad\langle\dot{x}(0), \ddot{x}(0)\rangle=0, \quad|\ddot{x}(0)|^{2}=-\langle\dot{x}(0), \dddot{x}(0)\rangle,
$$

where $\langle\cdot, \cdot\rangle$ is the $\mathbb{R}^{2}$ scalar product and $|\cdot|$ is the Euclidean norm.

Proof. - We have that

$$
g(\dot{x}(t), \dot{x}(t))=1 .
$$

and since $g(0)=\mathrm{Id}$, we deduce that $|\dot{x}(0)|=1$. Next, we differentiate (4.10) with respect to $t$ which gives

$$
2 \sum_{i, j=1}^{2} g_{i j}(x(t)) \ddot{x}_{i}(t) \dot{x}_{j}(t)+\sum_{i, j, k=1}^{2} \frac{\partial g_{i j}}{\partial x_{k}}(x(t)) \dot{x}_{i}(t) \dot{x}_{j}(t) \dot{x}_{k}(t)=0 .
$$

Since we are working in geodesic coordinates the first derivatives of the metric are vanishing at the origin. Therefore by taking $t=0$ in (4.11), we obtain that 
$\langle\dot{x}(0), \ddot{x}(0)\rangle=0$. Since we are working in a geodesic coordinate system, we have the identity

$$
\sum_{j=1}^{2} g_{i j}(x(t)) x_{j}(t)=\sum_{j=1}^{2} g_{i j}(0) x_{j}(t), \quad i=1,2 .
$$

Differentiating three times (4.12) with respect to $t$ and taking $t=0$ gives

$$
\sum_{j, k, s=1}^{2} \frac{\partial^{2} g_{i j}}{\partial x_{k} \partial x_{s}}(0) \dot{x}_{k}(0) \dot{x}_{j}(0) \dot{x}_{s}(0)=0, \quad i=1,2 .
$$

Therefore differentiating (4.11) with respect to $t$ and taking $t=0$ gives

$$
|\ddot{x}(0)|^{2}=-\langle\dot{x}(0), \dddot{x}(0)\rangle \text {. }
$$

This completes the proof of Lemma 4.3 .

Next, we state an elementary lemma.

Lemma 4.4. - Let

$$
x(t)=\left(x_{1}(t), x_{2}(t)\right), t \sim 0
$$

be a smooth curve in $\mathbb{R}^{2}$ such that

$$
x(0)=0, \quad|\dot{x}(0)|=1, \quad\langle\dot{x}(0), \ddot{x}(0)\rangle=0, \quad|\ddot{x}(0)|^{2}=-\langle\dot{x}(0), \dddot{x}(0)\rangle,
$$

Then

$$
|x(t)|^{2}=t^{2}\left(1-\frac{t^{2}}{12}|\ddot{x}(0)|^{2}+\mathcal{O}\left(|t|^{3}\right)\right) .
$$

Proof. - Write the Taylor expansion

$$
x(t)=t\left(\dot{x}(0)+\frac{t}{2} \ddot{x}(0)+\frac{t^{2}}{6} \dddot{x}(0)+\mathcal{O}\left(|t|^{3}\right)\right) .
$$

We thus can write

$$
|x(t)|^{2}=t^{2}\left(|\dot{x}(0)|^{2}+t\langle\dot{x}(0), \ddot{x}(0)\rangle+\frac{t^{2}}{4}|\ddot{x}(0)|^{2}+\frac{t^{2}}{3}\langle\dot{x}(0), \dddot{x}(0)\rangle+\mathcal{O}\left(|t|^{3}\right)\right)
$$

which, taking into account (4.13), clearly yields (4.14). This completes the proof of Lemma 4.4.

With Lemma 4.4 in hand, we can now give a precise description of $\psi(\gamma(t), \gamma(\tau))$.

Lemma 4.5. - For $\varepsilon \ll 1, \gamma(t), \gamma(\tau) \in U$, we can write

$$
\psi(\gamma(t), \gamma(\tau))=-|t-\tau|\left(1-c(\tau)(t-\tau)^{2}+d(\tau, t-\tau)(t-\tau)^{3}\right),
$$

where $c(\tau)$ and $d(\tau, t-\tau)$ are smooth functions such that $c(\tau) \geq c>0$. 
Proof. - Let us consider a geodesic coordinate system $x=\left(x_{1}, x_{2}\right)$ centered at $\gamma(\tau)$. In this system the curve $\gamma$ is given by $\{x(s), s \sim 0\}$ with $x(0)=0$. Observe that

$$
|x(t-\tau)|=-\psi(\gamma(t), \gamma(\tau))
$$

and therefore we can apply Lemma 4.3 and Lemma 4.4 to conclude that

$$
\psi^{2}(\gamma(t), \gamma(\tau))=|t-\tau|^{2}\left(1-\frac{(t-\tau)^{2}}{12}|\ddot{x}(0)|^{2}+\mathcal{O}\left(|t-\tau|^{3}\right)\right) .
$$

We have that in the considered coordinate system

$$
\left.\gamma^{\prime}\right|_{x(s)}=\sum_{j=1}^{2} \dot{x}_{j}(s)\left(\frac{\partial}{\partial x_{j}}\right)_{x(s)}
$$

and by the definition of the covariant derivative along $\gamma$ (see e.g. [6]), we can write in the coordinates $\left(x_{1}, x_{2}\right)$,

$$
\left.\frac{D}{d t} \gamma^{\prime}\right|_{x(s)}=\sum_{i=1}^{2}\left(\ddot{x}_{i}(s)+\sum_{j, k=1}^{2} \Gamma_{j k}^{i}(x(s)) \dot{x_{j}}(s) \dot{x_{k}}(s)\right)\left(\frac{\partial}{\partial x_{i}}\right)_{x(s)},
$$

where $\Gamma_{j k}^{i}$ are the Christoffel symbols of the metric $g$. Since we are working in a geodesic coordinate system, we have that $\Gamma_{j k}^{i}(0)=0$. Therefore

$$
|\ddot{x}(0)|^{2}=g\left(\frac{D}{d t} \gamma^{\prime}(\tau), \frac{D}{d t} \gamma^{\prime}(\tau)\right)=24 c(\tau) \neq 0
$$

thanks to the assumption (1.3) on the curve $\gamma$. Consequently, coming back to (4.15) and using that $\psi$ is non-positive, we obtain the assertion of Lemma 4.5 .

In view Lemma 4.1 and Lemma 4.5] it is now clear that (1.4) is a consequence of the following oscillatory integral lemma.

Lemma 4.6. - Let $\chi \in C_{0}^{\infty}$ satisfying $\sqrt{4.8)}$, $a \in C_{0}^{\infty}\left(\mathbb{R}^{2}\right)$ with support $\mathcal{O}(\varepsilon)$, $\varepsilon>0$. Let $\gamma \in C^{\infty}\left(\mathbb{R} \times \mathbb{R}^{\star}\right)$ be of the form

$$
\gamma(\tau, t-\tau)= \pm|t-\tau|\left(1-c(\tau)(t-\tau)^{2}+d(\tau, t-\tau)(t-\tau)^{3}\right),
$$

where $c(\tau)$ and $d(\tau, t-\tau)$ are smooth functions such that $c(\tau) \geq c>0$. We define the linear map

$$
\left(K_{\lambda} f\right)(t)=\int_{-\infty}^{\infty} e^{i \lambda \gamma(\tau, t-\tau)} \frac{a(\tau, t-\tau)}{(\lambda|t-\tau|)^{\frac{1}{2}}}(1-\chi)\left(\lambda^{1 / 3}(t-\tau)\right) f(\tau) d \tau .
$$

Then for $\varepsilon \ll 1$,

$$
\left\|K_{\lambda} f\right\|_{L^{2}} \leq C \lambda^{-\frac{2}{3}}\|f\|_{L^{2}} .
$$


Proof. - As a startup and to enlighten the exposition below, let us first indicate how to prove the result in the model case

$$
\gamma(\tau, t-\tau)=(t-\tau)+c(t-\tau)^{3}, \quad a(\tau, t-\tau)=1
$$

In that case our operator is (modulo a conjugation by $e^{i \lambda s}$ which is an isometry on $\left.L^{2}\right)$ a convolution operator with convolution kernel

$$
k(r)=e^{i \lambda r^{3}} \frac{(1-\chi)\left(\lambda^{1 / 3} r\right)}{\lambda|r|^{1 / 2}}
$$

and consequently the norm of the operator is equal to

$$
\|\widehat{k}\|_{L^{\infty}}=\lambda^{-2 / 3} \sup _{\rho}|\widehat{\widetilde{k}}|\left(\lambda^{-1 / 3} \rho\right)=\lambda^{-2 / 3} \sup _{\rho}|\widehat{\widetilde{k}}|(\rho)
$$

with

$$
\widetilde{k}(r)=e^{i r^{3}} \frac{(1-\chi)(r)}{|r|^{1 / 2}}
$$

having a bounded Fourier transform, as can be seen by a straightforward application of van der Corput lemma.

We now come back to the general case. We shall only consider the case when the sign in front of $|t-\tau|$ in the definition of $\gamma(\tau, t-\tau)$ is minus, the case of sign plus being similar. We split the kernel of $K_{\lambda}$ into two parts supported respectively in the sets $\{t \geq \tau\}$ and $\{t \leq \tau\}$ which yields

$$
K_{\lambda}=K_{\lambda}^{+}+K_{\lambda}^{-} .
$$

With

$$
\gamma_{1}(\tau, t-\tau)=\gamma(\tau, t-\tau)+|t-\tau|=c(\tau)(t-\tau)^{3}-d(\tau, t-\tau)(t-\tau)^{4},
$$

we can write

$$
\left(K_{\lambda}^{+} f\right)(t)=e^{-i \lambda t} \int_{t \geq \tau} e^{i \lambda \gamma_{1}(\tau, t-\tau)} \frac{a(\tau, t-\tau)}{(\lambda|t-\tau|)^{\frac{1}{2}}}(1-\chi)\left(\lambda^{1 / 3}(t-\tau)\right) e^{i \tau \lambda} f(\tau) d \tau .
$$

It is now clear that it suffices to establish the estimate

$$
\left\|\widetilde{K}_{\lambda}^{+} f\right\|_{L^{2}} \leq C \lambda^{-\frac{2}{3}}\|f\|_{L^{2}}
$$

where

$$
\left(\widetilde{K}_{\lambda}^{+} f\right)(t)=\int_{t \geq \tau} e^{i \lambda \gamma_{1}(\tau, t-\tau)} \frac{a(\tau, t-\tau)}{(\lambda|t-\tau|)^{\frac{1}{2}}}(1-\chi)\left(\lambda^{1 / 3}(t-\tau)\right) f(\tau) d \tau .
$$


Let us compute the Fourier transform

$\widehat{\widetilde{K}_{\lambda}^{+} f}(\xi)=\int_{-\infty}^{\infty} \int_{t \geq \tau} \exp \left(i \lambda \gamma_{1}(\tau, t-\tau)-i t \xi\right) \frac{a(\tau, t-\tau)}{(\lambda|t-\tau|)^{\frac{1}{2}}}(1-\chi)\left(\lambda^{1 / 3}(t-\tau)\right) f(\tau) d \tau d t$.

We now exchange the order of integration and then we perform a change of variables $(\tau, t) \rightarrow(\tau, z)$ where $t=\tau+z / \lambda^{1 / 3}$. Therefore

$$
\begin{aligned}
& \widehat{\widetilde{K}_{\lambda}^{+} f}(\xi) \\
= & \lambda^{-\frac{2}{3}} \int_{-\infty}^{\infty} \int_{1 / 2}^{\infty} \exp \left(i \lambda \gamma_{1}\left(\tau, \frac{z}{\lambda^{1 / 3}}\right)-i \tau \xi-i \frac{z \xi}{\lambda^{1 / 3}}\right) a\left(\tau, \frac{z}{\lambda^{1 / 3}}\right) \tilde{\chi}(z) f(\tau) d z d \tau
\end{aligned}
$$

where

$$
\widetilde{\chi}(z)=\frac{(1-\chi)(z)}{z^{1 / 2}}
$$

We can therefore write

$$
\widehat{\widetilde{K}_{\lambda}^{+} f}(\xi)=\lambda^{-\frac{2}{3}} \mathcal{K} f(\xi)
$$

where

$$
\mathcal{K} f(\xi)=\int_{-\infty}^{\infty} e^{-i \tau \xi} A_{\lambda}(\tau, \xi) f(\tau) d \tau
$$

with

$$
A_{\lambda}(\tau, \xi)=\int_{1 / 2}^{\infty} \exp \left(i\left[\lambda \gamma_{1}\left(\tau, \frac{z}{\lambda^{1 / 3}}\right)-\frac{\xi z}{\lambda^{1 / 3}}\right]\right) a\left(\tau, \frac{z}{\lambda^{1 / 3}}\right) \tilde{\chi}(z) d z .
$$

We therefore reduced the matters to establishing a uniform $L^{2}$ bound on the linear map $\mathcal{K}$. Let us denote by $\Phi_{\lambda}(\tau, \xi, z)$ the phase function in the definition of $A_{\lambda}(\tau, \xi)$, i.e.

$$
\Phi_{\lambda}(\tau, \xi, z)=\lambda \gamma_{1}\left(\tau, \frac{z}{\lambda^{1 / 3}}\right)-\frac{\xi z}{\lambda^{1 / 3}} .
$$

Coming back to the definition of $\gamma_{1}$, we can write

$$
\Phi_{\lambda}(\tau, \xi, z)=c(\tau) z^{3}-\xi \frac{z}{\lambda^{1 / 3}}-d\left(\tau, \frac{z}{\lambda^{1 / 3}}\right) \frac{z^{4}}{\lambda^{1 / 3}} .
$$

Hence

$$
\frac{\partial \Phi_{\lambda}}{\partial z}(\tau, \xi, z)=3 c(\tau) z^{2}-\frac{\xi}{\lambda^{1 / 3}}-d\left(\tau, \frac{z}{\lambda^{1 / 3}}\right) \frac{z}{\lambda^{1 / 3}} 4 z^{2}-\frac{\partial d}{\partial z}\left(\tau, \frac{z}{\lambda^{1 / 3}}\right)\left(\frac{z}{\lambda^{1 / 3}}\right)^{2} z^{2} .
$$

Therefore for $\varepsilon \ll 1$ and $(z, \tau)$ on the support of $a\left(\tau, \frac{z}{\lambda^{1 / 3}}\right)$

$$
\left|\frac{\partial \Phi_{\lambda}}{\partial z}(\tau, \xi, z)\right| \geq c z^{2}
$$


provided $\xi \leq 0$. On the other hand for every $\xi \in \mathbb{R}$, every $k \geq 2$, every $(z, \tau)$ on the support of $a\left(\tau, \frac{z}{\lambda^{1 / 3}}\right)$, we have the bound

$$
\left|\frac{\partial^{k} \Phi_{\lambda}}{\partial z^{k}}(\tau, \xi, z)\right| \leq C z^{\max (3-k, 0)} .
$$

Therefore, we can integrate by parts with the operator

$$
\frac{1}{i \frac{\partial \Phi_{\lambda}}{\partial z}} \frac{\partial}{\partial z} .
$$

Each such integration by parts gains $\frac{1}{c z^{3}}$. Since derivatives with respect to $\tau$ give only (fixed) powers of $z$, we obtain that for $\xi \leq 0, k=0,1, \ldots$,

$$
\left|\partial_{\tau}^{k} A_{\lambda}(\tau, \xi)\right| \leq C_{k}
$$

Notice that if $|\xi| \leq C \lambda^{1 / 3}$ then we can put the slowly oscillating factor $e^{-i \xi z / \lambda^{1 / 3}}$ in the amplitude $\tilde{\chi}(z)$, and, the new phase satisfies the bound (4.19) and similar integration by parts gaining now $\frac{1}{c z^{2}}$, we can still achieve the bound (4.21).

We obtain that for every $\Lambda>0$, every $k=0,1, \ldots$ there exists $C>0$ such that for every $\xi \leq \Lambda \lambda^{1 / 3}$,

$$
\left|\partial_{\tau}^{k} A_{\lambda}(\tau, \xi)\right| \leq C
$$

Let $\chi_{0} \in C_{0}^{\infty}(\mathbb{R})$ be such that

$$
\chi_{0}(x)= \begin{cases}0, & |x| \leq 1 / 2 \\ 1, & |x| \geq 1\end{cases}
$$

For $\Lambda>0$ to be fixed later, we consider the splitting

$$
\mathcal{K}=\mathcal{K}_{1}+\mathcal{K}_{2},
$$

where

$$
\left(\mathcal{K}_{1} f\right)(\xi)=\left(1-\chi_{0}\right)\left(\frac{\xi}{\Lambda \lambda^{1 / 3}}\right)(\mathcal{K} f)(\xi)
$$

Observe that

$$
\left(\mathcal{K}_{1} f\right)(\xi)=\int_{-\infty}^{\infty}\left(1-\chi_{0}\right)\left(\frac{\xi}{\Lambda \lambda^{1 / 3}}\right) \widehat{A_{\lambda}}(\xi-\sigma, \xi) \hat{f}(\sigma) \frac{d \sigma}{2 \pi}
$$

where $\widehat{A_{\lambda}}$ denotes the Fourier transform of $A_{\lambda}$ with respect to the first variable. Thanks to (4.22), and using the compactness of the support of $A_{\lambda}(\tau, \xi)$ with respect to $\tau$, we obtain that for every $N \in \mathbb{N}$ there exist $C_{N}$ such that

$$
\left|\left(1-\chi_{0}\right)\left(\frac{\xi}{\Lambda \lambda^{1 / 3}}\right) \widehat{A_{\lambda}}(\xi-\sigma, \xi)\right| \leq \frac{C_{N}}{(1+|\xi-\sigma|)^{N}} .
$$


Estimate (4.23) with $N=2$ and the Young inequality imply the uniform (with respect to $\lambda$ ) boundedness of $\mathcal{K}_{1}$ on $L^{2}(\mathbb{R})$.

It remains to estimate $\mathcal{K}_{2}$ as an operator on $L^{2}(\mathbb{R})$. For $\xi>0$, we consider a change of variable

$$
z \mapsto\left(\frac{\xi}{\lambda^{1 / 3}}\right)^{1 / 2} z
$$

which allows to write

$$
A_{\lambda}(\tau, \xi)=\left(\frac{\xi}{\lambda^{1 / 3}}\right)^{\frac{1}{2}} \int \exp \left(i\left(\frac{\xi}{\lambda^{1 / 3}}\right)^{\frac{3}{2}} \phi_{\lambda}(\tau, \xi, z)\right) a\left(\tau, \frac{\sqrt{\xi} z}{\sqrt{\lambda}}\right) \widetilde{\chi}\left(\frac{\sqrt{\xi} z}{\lambda^{1 / 6}}\right) d z .
$$

Remark that due to support considerations, in the integral above

$$
z \geq \frac{1}{2}\left(\frac{\xi}{\lambda^{1 / 3}}\right)^{-\frac{1}{2}}
$$

and

$$
\phi_{\lambda}(\tau, \xi, z)=c(\tau) z^{3}-z-d\left(\tau, \frac{\sqrt{\xi} z}{\sqrt{\lambda}}\right) \frac{\sqrt{\xi} z}{\sqrt{\lambda}} z^{3}
$$

Now we set

$$
\omega=\left(\frac{\xi}{\lambda^{1 / 3}}\right)^{3 / 2}
$$

and we intend to apply the stationary phase formula ( $\omega$ being the large parameter) to the integral (4.24). Consider the equation

$$
3 c(\tau) z^{2}-1+\mathcal{O}\left(\varepsilon z^{2}\right)=0
$$

satisfied by the critical points with respect to $z$ of the phase $\phi_{\lambda}(\tau, \xi, z)$ on the support of $a\left(\tau, \frac{\sqrt{\xi} z}{\sqrt{\lambda}}\right)$. For $\varepsilon \ll 1$, the equation (4.26) has a unique positive root $z=z_{\lambda}(\tau, \xi)$ which satisfies

$$
0<c_{1} \leq z_{\lambda}(\tau, \xi) \leq c_{2} .
$$

In addition, the critical point $z_{\lambda}(\tau, \xi)$ is non degenerate

$$
\exists c>0 ; c \leq \frac{\partial^{2} \phi_{\lambda}}{\partial z^{2}}\left(z_{\lambda}(\tau, \xi)\right) \leq \frac{1}{c} .
$$

Since

$$
\frac{\partial \phi_{\lambda}}{\partial z}\left(\tau, \xi, z_{\lambda}(\tau, \xi)\right)=0
$$

Differentiating (4.28) with respect to $\tau$ gives

$$
\frac{\partial^{2} \phi_{\lambda}}{\partial z \partial \tau}\left(\tau, \xi, z_{\lambda}(\tau, \xi)\right)+\frac{\partial z_{\lambda}}{\partial \tau}(\tau, \xi) \frac{\partial^{2} \phi_{\lambda}}{\partial z^{2}}\left(\tau, \xi, z_{\lambda}(\tau, \xi)\right)=0 .
$$


Coming back to (4.25) and using (4.27), we obtain that

$$
\left|\frac{\partial z_{\lambda}}{\partial \tau}(\tau, \xi)\right| \leq C \sqrt{\frac{\xi}{\lambda}}
$$

Similarly, differentiating (4.29) with respect to $\tau$, we get for $k \geq 1$,

$$
\left|\frac{\partial^{k} z_{\lambda}}{\partial \tau^{k}}(\tau, \xi)\right| \leq C_{k} .
$$

Next, we notice that

$$
\left|\left(\frac{\partial}{\partial \tau}\right)^{p}\left(\frac{\partial}{\partial z}\right)^{q} a\left(\tau, \frac{\sqrt{\xi} z}{\sqrt{\lambda}}\right) \widetilde{\chi}\left(\frac{\sqrt{\xi} z}{\lambda^{1 / 6}}\right)\right| \leq C_{p, q}\left(\frac{\xi}{\lambda^{1 / 3}}\right)^{\frac{q}{2}} .
$$

But for $\omega \gg 1$ we have that

$$
\left(\frac{\xi}{\lambda^{1 / 3}}\right)^{q} \ll \omega^{q}
$$

Therefore, for $\xi / \lambda \leq C$, we can apply the stationary phase formula to sufficiently high order (we recall that in the expansion given by the stationary phase formula the $k$-th term in $\omega^{-k}$ involves derivatives of order $2 k$ of the symbol, see [11, Th. 7.7.1]), and we get

$$
A_{\lambda}(\tau, \xi)=\exp \left(i \omega V_{\lambda}(\tau, \xi)\right) q_{\lambda}(\tau, \xi)
$$

where $q_{\lambda}(\tau, \xi)$ satisfies

$$
\left|\partial_{\tau}^{k} q_{\lambda}(\tau, \xi)\right| \leq C_{k}\left(\frac{\xi}{\lambda^{1 / 3}}\right)^{-\frac{1}{4}}, \quad k=0,1,2,3
$$

and $V_{\lambda}(\tau, \xi)$ is the value of the phase $\phi_{\lambda}(\tau, \xi, z)$ at the critical point $z=z_{\lambda}(\tau, \xi)$, hence satisfies

$$
\left|\partial_{\tau}^{k} V_{\lambda}(\tau, \xi)\right| \leq C_{k}, \quad k=0,1,2,3 .
$$

Moreover, if $\frac{\xi}{\lambda} \gg 1$, then the critical point $z_{\lambda}(\tau, \xi)$ lies far from the support of the function $a$ and consequently, by integrating by parts and using (4.20) (4.31), we obtain a contribution rapidly decaying with respect to $\lambda$. Observe that the condition $\omega \gg 1$ can be achieved by taking $\Lambda \gg 1$ in the definition of the cut-off $\chi_{0}$.

Let $\chi_{1} \in C_{0}^{\infty}(\mathbb{R})$ be equal to one in a neighborhood of zero. Consider the splitting

$$
\mathcal{K}_{2}=\mathcal{K}_{21}+\mathcal{K}_{22}
$$

where

$$
\left(\mathcal{K}_{21} f\right)(\xi)=\left(1-\chi_{1}\right)\left(\frac{\xi}{\delta \lambda}\right)\left(\mathcal{K}_{2} f\right)(\xi) .
$$

Notice that for $\xi$ on the support of $\left(1-\chi_{1}\right)\left(\frac{\xi}{\delta \lambda}\right)$ one has

$$
|\xi| \gtrsim \delta \lambda \text {. }
$$


On the other hand, on the domain of integration in (4.24),

$$
\left|\frac{\sqrt{\xi} z}{\sqrt{\lambda}}\right| \lesssim \varepsilon
$$

Therefore, if $\delta=\kappa \varepsilon^{2}$ with $\kappa$ large enough, we obtain that (4.33) and (4.34) imply $|z| \leq c_{1} / 2$. Hence, in view of (4.27), for $\xi$ on the support of $\left(1-\chi_{1}\right)\left(\frac{\xi}{\delta \lambda}\right)$ the phase function $\phi_{\lambda}(\tau, \xi, z)$ has no critical point in $z$ and we can evaluate $\mathcal{K}_{21}$ as we did for $\mathcal{K}_{1}$ thanks to the rapid decay (in $\lambda$ ) satisfied by $A_{\lambda}(\tau, \xi)$.

It remains to deal with $\mathcal{K}_{22}$. For that purpose, we make another appeal to a duality argument. We need to establish the uniform (with respect to $\lambda$ ) boundedness on $L^{2}(\mathbb{R})$ of the operator

$$
\left(\mathcal{K}_{22} f\right)(\xi)=\chi_{0}\left(\frac{\xi}{\Lambda \lambda^{1 / 3}}\right) \chi_{1}\left(\frac{\xi}{\delta \lambda}\right) \int_{-\infty}^{\infty} e^{-i \tau \xi} e^{i \omega V_{\lambda}(\tau, \xi)} q_{\lambda}(\tau, \xi) f(\tau) d \tau .
$$

By duality one therefore needs to study the uniform $L^{2}$ boundedness of $\mathcal{K}_{22} \mathcal{K}_{22}^{\star}$, where $\mathcal{K}_{22}^{\star}$ is the formal adjoint of $\mathcal{K}_{22}$ with respect to the $L^{2}(\mathbb{R})$ inner product. Write

$$
\left(\mathcal{K}_{22} \mathcal{K}_{22}^{\star} f\right)(\xi)=\int_{-\infty}^{\infty} S(\xi, \sigma) f(\sigma) d \sigma
$$

where

$$
\begin{aligned}
S(\xi, \sigma)= & \chi_{0}\left(\frac{\xi}{\Lambda \lambda^{1 / 3}}\right) \chi_{1}\left(\frac{\xi}{\delta \lambda}\right) \chi_{0}\left(\frac{\sigma}{\Lambda \lambda^{1 / 3}}\right) \chi_{1}\left(\frac{\sigma}{\delta \lambda}\right) \times \\
& \times \int_{-\infty}^{\infty} e^{-i \tau(\xi-\sigma)} \exp \left(i\left[\omega V_{\lambda}(\tau, \xi)-\frac{\sigma^{3 / 2}}{\lambda^{1 / 2}} V_{\lambda}(\tau, \sigma)\right]\right) q_{\lambda}(\tau, \xi) \overline{q_{\lambda}}(\tau, \sigma) d \tau
\end{aligned}
$$

We next write

$$
\omega V_{\lambda}(\tau, \xi)-\frac{\sigma^{3 / 2}}{\lambda^{1 / 2}} V_{\lambda}(\tau, \sigma)=\frac{\xi^{3 / 2}}{\lambda^{1 / 2}} V_{\lambda}(\tau, \xi)-\frac{\sigma^{3 / 2}}{\lambda^{1 / 2}} V_{\lambda}(\tau, \sigma)=(\xi-\sigma) R_{\lambda}(\tau, \xi, \sigma) .
$$

The relevant fact about $R_{\lambda}$ is that for $\varepsilon \ll 1$, the map

$$
\tau \mapsto \tau-R_{\lambda}(\tau, \xi, \sigma)
$$

is a small perturbation of the identity and thus the corresponding integral operator is $L^{2}$ bounded. Let us now give the precise argument.

$$
V_{\lambda}(\tau, \xi)=\phi_{\lambda}\left(\tau, \xi, z_{\lambda}(\tau, \xi)\right)
$$

and therefore, by invoking (4.28), we obtain that

$$
\frac{\partial V_{\lambda}}{\partial \xi}(\tau, \xi)=\frac{\partial \phi_{\lambda}}{\partial \xi}\left(\tau, \xi, z_{\lambda}(\tau, \xi)\right) .
$$


Coming back to the definition of $\phi_{\lambda}$, using (4.27) and (4.30), we obtain that

$$
\left|\left(\frac{\partial}{\partial \tau}\right)^{k} \frac{\partial V_{\lambda}}{\partial \xi}(\tau, \xi)\right| \leq C_{k} \xi^{-\frac{1}{2}} \lambda^{-\frac{1}{2}}
$$

Since $\delta \lesssim \epsilon^{2}$, estimates (4.32) and (4.36) imply the bound

$$
\left|\left(\frac{\partial}{\partial \tau}\right)^{k}\left(\frac{\partial}{\partial \xi}\right)\left[\frac{\xi^{3 / 2}}{\lambda^{1 / 2}} V_{\lambda}(\tau, \xi)\right]\right| \leq C\left(\sqrt{\frac{\xi}{\lambda}}+\frac{\xi}{\lambda}\right) \leq C \varepsilon, \quad k=0,1,2,3
$$

for $\xi$ on the support of $\chi_{1}\left(\frac{\xi}{\delta \lambda}\right)$. Coming back to (4.35), we obtain

$$
\left|\partial_{\tau}^{k} R_{\lambda}(\tau, \xi, \tau)\right| \leq C \varepsilon, \quad k=0,1,2,3 .
$$

For $\varepsilon \ll 1$, after two integrations by parts in $\tau$, we obtain the bound

$$
|S(\xi, \sigma)| \leq \frac{C}{(1+|\xi-\sigma|)^{2}}
$$

and therefore using the Young inequality we obtain that $\mathcal{K}_{22} \mathcal{K}_{22}^{\star}$ is bounded on $L^{2}(\mathbb{R})$, uniformly in $\lambda$. This completes the proof of Lemma 4.6] and consequently of (1.4).

\section{On the optimality of (1.1) and (1.4)}

5.1. Optimality for the spectral projectors on any manifold. - In this section we prove lower bounds for the smoothed spectral projectors $\chi_{\lambda}$ involved in Theorem 3. In section 5.2 we will use these bounds to get the optimality of (1.1) and (1.4) in the particular case of the standard sphere. Let us first study the case $p \geq 4$.

Lemma 5.1. - Let $p \geq 4$. For any smooth curve $\gamma$, there exists $c>0$ such that for any $\lambda \geq 1$,

$$
\left\|\chi_{\lambda}\right\|_{\left.\mathcal{L}\left(L^{2}(M) ; L^{p}(\gamma)\right)\right)} \geq c \lambda^{\frac{1}{2}-\frac{1}{p}}
$$

Proof. - Indeed since

$$
\left\|\chi_{\lambda}\right\|_{\left.\mathcal{L}\left(L^{2}(M) ; L^{p}(\gamma)\right)\right)}^{2}=\left\|\chi_{\lambda} \chi_{\lambda}^{*}\right\|_{\left.\mathcal{L}\left(L^{p^{\prime}}(\gamma) ; L^{p}(\gamma)\right)\right)}
$$

and since

it suffices to prove

$$
\left\|R_{\lambda}\right\|_{\left.\mathcal{L}\left(L^{2}(M) ; L^{p}(\gamma)\right)\right)} \leq C \lambda^{-1 / 2},
$$

$$
\left\|T_{\lambda} T_{\lambda}^{\star}\right\|_{\mathcal{L}\left(L^{p^{\prime}}(\gamma) ; L^{p}(\gamma)\right)} \geq c \lambda^{-\frac{2}{p}} .
$$


But if $|t-s| \leq 2 \epsilon \lambda^{-1}$ then the oscillatory factor $e^{i \lambda[\psi(x(t), y)-\psi(x(s), y)]}$ does not oscillate any more and if $\epsilon>0$ is chosen small enough, the kernel $K$ of the operator $T_{\lambda} T_{\lambda}^{\star}$ satisfies

$$
\begin{aligned}
\operatorname{Re}(K(t, s))=\operatorname{Re}(\mathcal{K}(x(t), x(s))) & \\
& =\int_{V} \operatorname{Re}\left(e^{i \lambda[\psi(x(t), y)-\psi(x(s), y)]}\right) a(x(t), y) a(x(s), y) d y \geq \alpha>0 .
\end{aligned}
$$

As a consequence, choosing as test functions $f(s)=\lambda^{\frac{1}{p^{\prime}}} \phi\left(\frac{\lambda s}{\epsilon}\right)$ (with $\phi \in C_{0}^{\infty}(-1,1)$ real and non negative), we obtain (for a constant $c>0$ )

$$
\begin{aligned}
\operatorname{Re}\left(1_{|t| \leq \epsilon \lambda^{-1}} T_{\lambda} T_{\lambda}^{\star} f\right) & \geq c 1_{|t| \leq \epsilon \lambda^{-1}} \int \lambda^{\frac{1}{p^{\prime}}} \phi\left(\frac{\lambda s}{\epsilon}\right) d s \\
& \geq c 1_{|t| \leq \epsilon \lambda^{-1}} \lambda^{\frac{1}{p^{\prime}}-1}
\end{aligned}
$$

and

$$
\left\|T_{\lambda} T_{\lambda}^{\star} f\right\|_{L^{p}} \geq c \lambda^{\frac{1}{p^{\prime}}-1-\frac{1}{p}}=c \lambda^{-\frac{2}{p}}
$$

We now turn to the case $2 \leq p \leq 4$ if $\gamma$ is a geodesic.

Lemma 5.2. - Let $2 \leq p \leq 4$. For any geodesic $\gamma$, there exist $c>0$ such that for any $\lambda \geq 1$,

$$
\left\|\chi_{\lambda}\right\|_{\left.\mathcal{L}\left(L^{2}(M) ; L^{p}(\gamma)\right)\right)} \geq c \lambda^{\frac{1}{4}}
$$

Proof. - As in the previous case, this lemma is equivalent to

$$
\left\|T_{\lambda} T_{\lambda}^{\star}\right\|_{\mathcal{L}\left(L^{p^{\prime}}(\gamma) ; L^{p}(\gamma)\right)} \geq c \lambda^{-\frac{1}{2}} .
$$

Assume that the geodesic is parametrized by arc length. The critical value of the phase in the expression of the kernel of the operator $T_{\lambda} T_{\lambda}^{\star}$ is

$$
\psi(x(t), x(s))=|t-s|
$$

and coming back to the expression of the kernel given by Lemma 4.1 we have for $C \lambda^{-1} \leq|t-s|$,

$$
\begin{aligned}
K(t, s)= & \mathcal{K}(x(t), x(s)) \\
& =e^{i \lambda|t-s|} \frac{a^{+}(t, s)}{(\lambda|t-s|)^{1 / 2}}+e^{-i \lambda|t-s|} \frac{a^{-}(t, s)}{(\lambda|t-s|)^{1 / 2}}+\mathcal{O}\left(\frac{1}{(\lambda|t-s|)^{3 / 2}}\right)
\end{aligned}
$$


where $a^{ \pm}$is non negative and does not vanish for $t, s$ in a neighborhood of $(0,0)$. As a consequence, choosing as test functions $f(s)=e^{i \lambda s} \phi\left(\frac{s-2 \epsilon}{\epsilon}\right)$ (with $\phi \in C_{0}^{\infty}(-1,1)$ real and non negative), we obtain

$$
\begin{aligned}
\operatorname{Re}\left(e^{-i \lambda t} 1_{4 \epsilon \leq t \leq 5 \epsilon} T_{\lambda} T_{\lambda}^{\star} f\right) & \\
\geq & 1_{4 \epsilon \leq t \leq 5 \epsilon} \operatorname{Re}\left(\int e^{-i \lambda t} K(t, s) e^{i \lambda s} \phi\left(\frac{s-2 \epsilon}{\epsilon}\right) d s\right) \\
\geq & 1_{4 \epsilon \leq t \leq 5 \epsilon} \int_{\epsilon \leq s \leq 2 \epsilon} \frac{a^{+}(t, s)}{(\lambda(t-s))^{1 / 2}} \phi\left(\frac{s-2 \epsilon}{\epsilon}\right) d s \\
& -\left|\int_{\epsilon \leq s \leq 2 \epsilon} e^{-2 i \lambda(t-s)} \frac{a^{-}(t, s)}{(\lambda(t-s))^{1 / 2}} \phi\left(\frac{s-2 \epsilon}{\epsilon}\right) d s\right|-\mathcal{O}\left(\lambda^{-3 / 2}\right)
\end{aligned}
$$

We can integrate by parts with respect to $s$ in the second integral in the right hand side of (5.4) and gain any power of $\lambda^{-1}$. As a consequence, the main contribution is the first term and we obtain

$$
\left\|T_{\lambda} T_{\lambda}^{\star} f\right\|_{L^{p}} \geq \frac{c}{\lambda^{1 / 2}} \quad(c>0)
$$

which completes the proof.

We finally turn to the case $2 \leq p \leq 4$ if $\gamma$ is geodesically curved.

Lemma 5.3. - Let $2 \leq p \leq 4$. For any geodically curved $\gamma$, there exists $c>0$ such that for any $\lambda \geq 1$,

$$
\left\|\chi_{\lambda}\right\|_{\left.\mathcal{L}\left(L^{2}(M) ; L^{p}(\gamma)\right)\right)} \geq c \lambda^{\frac{1}{3}-\frac{1}{3 p}}
$$

Proof. - As before this lemma is equivalent to

$$
\left\|T_{\lambda} T_{\lambda}^{\star}\right\|_{\mathcal{L}\left(L^{p^{\prime}}(\gamma) ; L^{p}(\gamma)\right)} \geq c \lambda^{-\frac{1}{3}-\frac{2}{3 p}} .
$$

In that case, we use the expression of the phase obtained in (4.15) and choose as test functions $f(s)=e^{i \lambda s} \lambda^{\frac{1}{3 p^{\prime}}} \phi\left(\frac{\lambda^{1 / 3} s-2 \epsilon}{\epsilon}\right)$ (with $\phi \in C_{0}^{\infty}(-1,1)$ real and non negative) and obtain

$$
\begin{aligned}
& \operatorname{Re}\left(e^{-i \lambda t} 1_{4 \epsilon \lambda^{-1 / 3} \leq t \leq 5 \epsilon \lambda^{-1 / 3}} T_{\lambda} T_{\lambda}^{\star} f\right) \\
& \quad \geq 1_{4 \epsilon \lambda^{-1 / 3} \leq t \leq 5 \epsilon \lambda^{-1 / 3}} \operatorname{Re}\left(\int e^{-i \lambda t} K(t, s) e^{i \lambda s} \lambda^{\frac{1}{3 p^{\prime}}} \phi\left(\frac{\lambda^{1 / 3} s-2 \epsilon}{\epsilon}\right) d s\right)
\end{aligned}
$$

But on the support in $s$ of the function $f$ and for $4 \epsilon \lambda^{-1 / 3} \leq t \leq 5 \epsilon \lambda^{-1 / 3}$, we have

$$
\epsilon \lambda^{-1 / 3} \leq s \leq 3 \epsilon \lambda^{-1 / 3} \leq 4 \epsilon \lambda^{-1 / 3} \leq t \leq 5 \epsilon \lambda^{-1 / 3}
$$


and consequently we can use the description of the kernel given in Lemma 4.1 and according to Lemma 4.5, for $\epsilon>0$ small enough

$$
\operatorname{Re}\left(e^{-i \lambda t} e^{-i \lambda \psi(t, s)} e^{i \lambda s}\right)=\operatorname{Re}\left(e^{-i c(s) \lambda(t-s)^{3}\left(1+\mathcal{O}\left(\lambda^{-1 / 3}\right)\right)}\right) \geq \frac{1}{2}
$$

We deduce, since $a^{+}(0,0)$ is positive,

$$
\begin{aligned}
& \operatorname{Re}\left(e^{-i \lambda t} 1_{4 \epsilon \lambda^{-1 / 3} \leq t \leq 5 \epsilon \lambda^{-1 / 3}} T_{\lambda} T_{\lambda}^{\star} f\right) \\
& \geq c 1_{4 \epsilon \lambda^{-1 / 3} \leq t \leq 5 \epsilon \lambda^{-1 / 3}} \int \phi\left(\frac{\lambda^{1 / 3} s-2 \epsilon}{\epsilon}\right) \times \frac{1}{(\lambda(t-s))^{1 / 2}} d s \\
& \quad-\left|\int e^{-i \lambda((t-s)+\psi(t, s))} \frac{a^{-}(t, s)}{(\lambda(t-s))^{1 / 2}} \phi\left(\frac{\lambda^{1 / 3} s-2 \epsilon}{\epsilon}\right) d s\right| \\
& \quad-\mathcal{O}\left(\lambda^{-1}\right) \int \phi\left(\frac{\lambda^{1 / 3} s-2 \epsilon}{\epsilon}\right) d s \\
& \geq c \lambda^{-1 / 3} 1_{4 \epsilon \lambda^{-1 / 3} \leq t \leq 5 \epsilon \lambda^{-1 / 3}} \int \phi\left(\frac{\lambda^{1 / 3} s-2 \epsilon}{\epsilon}\right) d s-\mathcal{O}\left(\lambda^{\frac{1}{3 p^{\prime}}-2}\right)
\end{aligned}
$$

where as in the previous case we integrated by parts with respect to the variable $s$ to gain powers of $\lambda^{-1}$ in the integral involving $a^{-}$(each such integration by parts gains $\lambda^{-1}$ and looses $\lambda^{1 / 3}$ due to the powers of $(t-s)$ ). As a consequence

$$
\left\|T_{\lambda} T_{\lambda}^{\star} f\right\|_{L^{p}} \geq c \lambda^{-\frac{2}{3}-\frac{1}{3 p}}=\lambda^{\frac{1}{3 p^{\prime}}-\frac{2}{3}-\frac{1}{3 p}}\|f\|_{L^{p^{\prime}}}=c \lambda^{-\frac{1}{3}-\frac{2}{3 p}}\|f\|_{L^{p^{\prime}}}
$$

which completes the proof.

\subsection{From spectral projectors to exact eigenfunctions on spheres. -}

Proof. - We first remark that our lower bound for the norm of the smoothed spectral projector $\chi_{\lambda}$ from $L^{2}(M)$ to $L^{p}(\gamma)$ gives a lower bound for the norm of the spectral projector

$$
\Pi_{\lambda}=1_{\sqrt{-\Delta}-\lambda \in\left[0, \frac{1}{2}\right)}
$$

in the following sense:

$$
\exists c>0, \exists \lambda_{n} \rightarrow+\infty, f_{n} \in L^{2}(M) ;\left\|\Pi_{\lambda_{n}} f_{n}\right\|_{L^{p}(\gamma)}>c \lambda_{n}^{\check{\delta}(p)}\left\|f_{n}\right\|_{L^{2}(M)}
$$

where $\check{\delta}(p)=\widetilde{\delta}(p)$ or $\check{\delta}(p)=\delta(p)$ according whether the curved is geodesically curved or not. Indeed, if the converse were true,

$$
\left\|\Pi_{\lambda} f\right\|_{L^{p}(\gamma)} \leq \varepsilon(\lambda) \lambda^{\check{\delta}(p)}\|f\|_{L^{2}(M)}, \quad \text { with } \lim _{\lambda \rightarrow+\infty} \varepsilon(\lambda)=0 .
$$


Writing a partition of unity

$$
1=\sum_{n \in \mathbb{Z}} \Pi_{\frac{n}{2}}
$$

and inserting another projector $\widetilde{\Pi}_{\lambda}=1_{\sqrt{-\Delta}-\lambda \in[-1,1]}$,

$$
\chi(\sqrt{-\Delta}-\lambda)=\sum_{n \in \mathbb{Z}} \Pi_{\frac{n}{2}} \widetilde{\Pi}_{\frac{n}{2}} \chi(\sqrt{-\Delta}-\lambda),
$$

we obtain

$$
\|\chi(\sqrt{-\Delta}-\lambda)\|_{\mathcal{L}\left(L^{2}(M) ; L^{p}(\gamma)\right)} \leq \sum_{n} \varepsilon(n) n^{\check{\delta}(p)}\left\|\widetilde{\Pi}_{\frac{n}{2}} \chi(\sqrt{-\Delta}-\lambda)\right\|_{\mathcal{L}\left(L^{2}(M)\right)} .
$$

But due to the rapid decay of the function $\chi$,

$$
\left\|\widetilde{\Pi}_{\frac{n}{2}} \chi(\sqrt{-\Delta}-\lambda)\right\|_{\mathcal{L}\left(L^{2}(M)\right)} \leq \frac{C_{N}}{\left(1+\left|\lambda-\frac{n}{2}\right|\right)^{N}}
$$

and consequently we obtain

$$
\|\chi(\sqrt{-\Delta}-\lambda)\|_{\mathcal{L}\left(L^{2}(M) ; L^{p}(\gamma)\right)} \leq o(1) \lambda^{\check{\delta}(p)} \quad \text { as } \lambda \rightarrow+\infty,
$$

which is contradicting our lower bounds for the spectral projector $\chi_{\lambda}$.

Now, if the manifold $M$ is a sphere, we know that $\Pi_{\lambda}$ has range either $\{0\}$ or the finite dimensional space or spherical harmonics of degree $k$ (corresponding to eigenvalues $-k(k+1)$ ) if $\sqrt{k(k+1)}-\lambda \in\left[0, \frac{1}{2}\right)$ (and in that case $k$ is uniquely determined). The sequence $\left(\lambda_{n}\right)$ appearing in (5.8) corresponds necessarily to that latter case and the sequence $g_{n}=\Pi_{\lambda_{n}} f_{n}$ satisfies the claimed lower bound.

Remark 5.4. - Inequality (5.1) is optimized on the sphere by the so called zonal spherical harmonics which concentrate in $\mathcal{O}\left(\lambda^{-1}\right)$ neighborhoods of two opposite points (the poles) and this can be easily seen by using asymptotic descriptions of these functions (see [22]). Inequality (5.2) is optimized by the highest weight spherical harmonics $H_{n}\left(x_{1}, x_{2}, x_{3}\right)=\left(x_{1}+i x_{2}\right)^{n}$ which concentrate on the equator $\left\{\left(x_{1}, x_{2}, x_{3}\right) \in\right.$ $\left.\mathbb{S}^{2} ; x_{3}=0\right\}$. Finally, inequality (5.5) is optimized for $p=2$ and if the curve is a parallel circle by some spherical harmonics which can be constructed by some turning point semi-classical analysis and concentrate on the parallel circles. In the general case (general curve or $2<p<4$ ), as suggested by one of the referees, one can check that the following spherical harmonics optimize inequality (5.5). Let $H_{n, \phi}$ be the spherical harmonic rotated by angle $\phi$ :

$$
H_{n, \phi}\left(x_{1}, x_{2}, x_{3}\right)=\left(x_{1}+i\left(\cos (\phi) x_{2}+\sin (\phi) x_{3}\right)\right)^{n}
$$


and for $\psi \in C_{0}^{\infty}(-1,1), \int \psi(s) d s=1$ and $\delta>0$ small enough

$$
u_{n}\left(x_{1}, x_{2}, x_{3}\right)=\int_{\phi} \Psi\left(\frac{N^{1 / 3} \phi}{\delta}\right) H_{n, \phi}\left(x_{1}, x_{2}, x_{3}\right) d \phi
$$

(Remark that $u_{n}$ is clearly a spherical harmonic)

\section{The higher dimensional case}

6.1. The estimates in Theorem 3, - In this section we will give the modifications required to handle the case of submanifolds of higher dimensional manifolds. Following the approach in Section 3, we define for

$$
z \sim 0 \in \mathbb{R}^{k} \rightarrow x(z) \sim 0 \in \mathbb{R}^{d}
$$

a system of coordinates on $\Sigma$,

$$
T_{\lambda}(f)=\mathcal{T}_{\lambda}(f)(x(z))
$$

and denote by $\mathcal{K}\left(x, x^{\prime}\right)$ the kernel of the operator $\mathcal{T}_{\lambda} \mathcal{T}_{\lambda}^{\star}$ and

$$
K\left(z, z^{\prime}\right)=\mathcal{K}\left(x(z), x\left(z^{\prime}\right)\right)
$$

the kernel of the operator $T_{\lambda} T_{\lambda}^{\star}$. The starting point of our analysis is the higher dimensional analog of Lemma 4.1

Lemma 6.1. - There exist $\varepsilon \ll 1,\left(a_{n}^{ \pm}, b_{n}\right)_{n \in \mathbb{N}} \in C^{\infty}\left(\mathbb{R}^{d} \times \mathbb{R}^{d} \times \mathbb{R}\right)$, such that for $\left\|x-x^{\prime}\right\| \gtrsim \lambda^{-1}$ and any $N \in \mathbb{N}^{*}$,

$$
\mathcal{K}\left(x, x^{\prime}\right)=\sum_{ \pm} \sum_{n=0}^{N-1} \frac{e^{ \pm i \lambda \psi\left(x, x^{\prime}\right)}}{\left(\lambda\left\|x-x^{\prime}\right\|\right)^{\frac{d-1}{2}+n}} a_{n}^{ \pm}\left(x, x^{\prime}, \lambda\right)+b_{N}\left(x, x^{\prime}, \lambda\right)
$$

where $\psi\left(x, x^{\prime}\right)$ is now the geodesic distance between the points $x$ and $x^{\prime}$. Moreover $a^{ \pm}$are real, have supports of size $\mathcal{O}(\varepsilon)$ with respect to the first two variables and are uniformly bounded with respect to $\lambda$. Finally

$$
\left|b_{N}\left(x, x^{\prime}, \lambda\right)\right| \leq C\left(\lambda\left|x-x^{\prime}\right|\right)^{-\frac{d-1}{2}-N} .
$$

The proof of this Lemma is essentially the same as the proof of Lemma 4.1 (see also [4. Lemma 2.7]. We simply apply the stationnary phase formula in its full strength (i.e. with the asymptotic expansion to order $P$, see for example 11, Theorem 7.7.5]), the two critical values of the phase $\left( \pm \psi\left(x, x^{\prime}\right)\right)$ being evaluated as in Section 4 .

We shall also use the following Young's and Hardy-Littlewood inequalities 
Lemma 6.2. - Consider $2 \leq p \leq+\infty$ and $T$ a convolution operator

$$
T f(x)=\int_{\mathbb{R}^{k}} K(x-y) f(y) d y .
$$

Then the norm of $T$ as an operator from $L^{p^{\prime}}\left(\mathbb{R}^{k}\right)$ to $L^{p}\left(\mathbb{R}^{k}\right)$ is bounded by $\|K\|_{L^{\frac{p}{2}}}$. Furthermore, if $K(x)=\frac{1}{|x|^{2 k / p}}$ and $p>2$, then the operator $T$ is still bounded from $L^{p^{\prime}}\left(\mathbb{R}^{k}\right)$ to $L^{p}\left(\mathbb{R}^{k}\right)$ (despite the logarithmic divergence of $\|K\|_{L^{\frac{p}{2}}\left(\mathbb{R}^{k}\right)}$ ).

To conclude the proof of Theorem 3 if $k<\frac{d-1}{2}$ is now easy: we simply remark that, since

$$
d_{g}\left(x(z), x\left(z^{\prime}\right)\right) \sim\left\|z-z^{\prime}\right\|,
$$

the kernel of $T_{\lambda}, K\left(x(z), x\left(z^{\prime}\right)\right)$ is bounded by

$$
C\left(1+\lambda\left\|z-z^{\prime}\right\|\right)^{-\frac{d-1}{2}}
$$

and apply the Young's inequality.

If $k<\frac{d-1}{2}$, we conclude that the operator $T_{\lambda} T_{\lambda}^{\star}$ is bounded from $L^{2}(\Sigma)$ to $L^{2}(\Sigma)$ by $C \lambda^{-k}$ (resp. from $L^{1}(\Sigma)$ to $L^{\infty}(\Sigma)$ by $C$ ) and consequently the operator $T_{\lambda}$ is bounded from $L^{2}(M)$ to $L^{2}(\Sigma)$ by $C \lambda^{-k / 2}$ (resp. from $L^{2}(M)$ to $L^{\infty}(\Sigma)$ by $C$ ). Taking into account that

$$
\varphi_{\lambda}=\lambda^{\frac{d-1}{2}} \mathcal{T}_{\lambda}\left(\varphi_{\lambda}\right)
$$

we obtain (if $k<\frac{d-1}{2}$ ) Theorem 3 for $p=2$ and $p=+\infty$ and consequently, by interpolation, for any $2 \leq p \leq+\infty$.

If $k \geq \frac{d-1}{2}$, the simple argument above still applies (using Hardy Littlewood inequality), but it gives Theorem 3 only in the range

$$
\begin{cases}p \geq \frac{4 k}{d-1} & \text { if } k>\frac{d-1}{2} \\ p>\frac{4 k}{d-1} & \text { if } k=\frac{d-1}{2} .\end{cases}
$$

To obtain the full result we have to refine the analysis and take advantage of some oscillations in the phase in (6.1). We first consider the contribution of $\left\{\left(z, z^{\prime}\right) ; \mid z-\right.$ $\left.z^{\prime} \mid \leq C \lambda^{-1}\right\}$ and define for $\chi \in C_{0}^{\infty}\left(\mathbb{R}^{d}\right)$

$$
K_{0}(z, z)=K(z, z) \chi\left(\lambda\left(z-z^{\prime}\right)\right) .
$$

Then, using that $K_{0}$ is bounded and supported in the set $\left\{\left(z, z^{\prime}\right) ;\left\|z-z^{\prime}\right\| \leq C \lambda^{-1}\right\}$, in view of Young's inequality, the contribution of $K_{0}$ is easily dealt with. Furthermore, 
the analysis above is sufficient, if $N$ is chosen large enough in Lemma 6.1 to deal with the contribution of the remainder term $b_{N}$. Indeed, if $N>\frac{d-1}{2}$

$$
\int_{\lambda^{-1} \leq|z| \leq 1} \frac{d z}{(\lambda|z|)^{\frac{d-1}{2}+N}} \leq C \lambda^{-k}
$$

As a consequence, we will focus on the contributions of the main term $a_{0}^{+}$and abusing notations we will still denote by $\mathcal{K}$ the function

$$
\mathcal{K}\left(x, x^{\prime}\right)=\frac{e^{i \lambda \psi\left(x, x^{\prime}\right)}}{\left(\lambda\left\|x-x^{\prime}\right\|\right)^{\frac{d-1}{2}}} a_{0}^{+}\left(x, x^{\prime}, \lambda\right)
$$

The contributions of the other terms $\left(a_{0}^{-}, a_{n}^{ \pm}, 1 \leq n<N\right)$ could be dealt with similarly, as will appear clearly in the proof below.

We now consider a partition of unity on $B=\left\{x \in \mathbb{R}^{k} ;|x|<1\right\}$,

$$
1=\chi(\lambda x)+\sum_{j=1}^{\log \lambda / \log 2} \tilde{\chi}\left(2^{j} x\right)
$$

where $\tilde{\chi} \in C_{0}^{\infty}\left(\mathbb{R}^{d}\right)$ and $\tilde{\chi}$ is supported in the set $\left\{x ; \frac{1}{2}<|x|<2\right\}$. We consider the related partition of the kernel:

$$
\begin{aligned}
K(z, z)=K(z, z) \chi\left(\lambda\left(z-z^{\prime}\right)\right)+\sum_{j=1}^{\log \lambda / \log 2} K\left(z, z^{\prime}\right) \widetilde{\chi}\left(2^{j}\left(z-z^{\prime}\right)\right) & \\
& \equiv K_{0}\left(z, z^{\prime}\right)+\sum_{j=1}^{\log \lambda / \log 2} K_{j}\left(z, z^{\prime}\right)
\end{aligned}
$$

The main step in the proof of Theorem 3 is the proof of

Proposition 6.3. - If $j$ is large enough, the operator $\left(T T^{\star}\right)_{j}$ whose kernel is $K_{j}\left(z, z^{\prime}\right)$ satisfies the bounds

$$
\begin{aligned}
\left\|\left(T T^{\star}\right)_{j} f\right\|_{L^{\infty}(\Sigma)} & \leq C\left(\frac{2^{j}}{\lambda}\right)^{\frac{d-1}{2}}\|f\|_{L^{1}(\Sigma)} \\
\left\|\left(T T^{\star}\right)_{j} f\right\|_{L^{2}(\Sigma)} & \leq C 2^{-j k}\left(\frac{2^{j}}{\lambda}\right)^{\frac{d-1}{2}+\frac{k-1}{2}}\|f\|_{L^{2}(\Sigma)}
\end{aligned}
$$

Let us first show how Proposition 6.3 implies Theorem 3 , by interpolation, we deduce

$$
\left\|\left(T T^{\star}\right)_{j} f\right\|_{L^{p}(\Sigma)} \leq C 2^{-\frac{2 j k}{p}}\left(\frac{2^{j}}{\lambda}\right)^{\frac{d-1}{2}+\frac{k-1}{p}}\|f\|_{L^{p^{\prime}}(\Sigma)}
$$


As a consequence, summing for $j \leq \log (\lambda) / \log (2)$ we obtain

$$
\begin{aligned}
\left\|T T^{\star} f\right\|_{\mathcal{L}\left(L^{p^{\prime}}(\Sigma) ; L^{p}(\Sigma)\right) \leq C \lambda^{-\frac{d-1}{2}-\frac{k-1}{p}} \sum_{j \leq \log (\lambda) / \log (2)} 2^{j\left(\frac{d-1}{2}-\frac{k+1}{p}\right)}} \\
\leq \begin{cases}C \lambda^{-\frac{d-1}{2}-\frac{k-1}{p}+\frac{d-1}{2}-\frac{k+1}{p}} & \text { if } p>\frac{2(k+1)}{d-1} \\
C \lambda^{-\frac{d-1}{2}-\frac{k-1}{p}} & \text { if } p<\frac{2(k+1)}{d-1} \\
C \lambda^{-\frac{d-1}{2}-\frac{k-1}{p}} \log (\lambda) & \text { if } p=\frac{2(k+1)}{d-1}\end{cases}
\end{aligned}
$$

which, taking (6.3) into account gives the estimates in Theorem 3 (remark that the case $p<2(k+1) /(d-1)$ occurs only when $k=d-1)$. Remark also that the fact that we can only prove the estimate for large $j$ is not a problem, as, shrinking the support of the symbol $a$ (i.e. taking $\epsilon$ small enough) in Theorem 4 , the contributions of small $j$ vanish.

We now come back to the proof of Proposition 6.3. The $L^{1}-L^{\infty}$ bound is straightforward and we can focus on the $L^{2}$ bound. We have

$$
K_{j}\left(z, z^{\prime}\right)=e^{i \lambda d\left(z, z^{\prime}\right)} \frac{\tilde{\chi}\left(2^{j}\left(z-z^{\prime}\right)\right)}{\left(\lambda d\left(z, z^{\prime}\right)\right)^{\frac{d-1}{2}}} a_{0}\left(z, z^{\prime}\right)
$$

where $d\left(z, z^{\prime}\right)$ is the geodesic distance (in $M$ ) between points $x(z)$ and $x\left(z^{\prime}\right)$. As a consequence, on the support of the r.h.s., $d\left(z, z^{\prime}\right) \sim 2^{-j}$. Next we introduce a partition of unity locally finite (uniformly with respect to $j$ ):

$$
1=\sum_{p \in \mathbb{Z}^{d-1}} \chi\left(2^{j} z-p\right)
$$

and write

$$
K_{j}\left(z, z^{\prime}\right)=\sum_{q, \widetilde{q} \in \mathbb{Z}^{d-1}} \chi\left(2^{j} z-q\right) K_{j}\left(z, z^{\prime}\right) \chi\left(2^{j} z^{\prime}-\widetilde{q}\right)
$$

We denote by $R_{j, q, \widetilde{q}}$ the operator whose kernel is

$$
\chi\left(2^{j} z-q\right) K_{j}\left(z, z^{\prime}\right) \chi\left(2^{j} z^{\prime}-\widetilde{q}\right) .
$$

Remark that due to the support properties of $K_{j}$, in the expression above, only the contributions from $(q, \widetilde{q})$ such that $\|q-\widetilde{q}\| \leq C$ do not vanish. Remark also that by quasi-orthogonality in $L^{2}$ (due to the fact that the partition of unity is locally finite), we have

$$
\left\|\left(T T^{\star}\right)_{j}\right\|_{\mathcal{L}\left(L^{2}(\Sigma)\right)} \leq C \sup _{q, \widetilde{q}}\left\|R_{j, q, \widetilde{q}}\right\|_{\mathcal{L}\left(L^{2}(\Sigma)\right)}
$$

For simplicity, we shall only estimate the norm of $R_{j, q, q}$ (the case of $(q, \widetilde{q}),\|q-\widetilde{q}\| \leq C$ is similar). Using a translation and an orthogonal (linear) transformation, we can 
assume that $q=0$ and the metric satisfies $g_{m, n}(q)=I d$. We now perform a change of variables, set $Z=2^{j} z$ and obtain

$$
\left\|R_{j, 0,0}\right\|_{\mathcal{L}\left(L^{2}(\Sigma)\right)}=2^{-j k}\left\|\underline{R_{j, 0,0}}\right\|_{\mathcal{L}\left(L^{2}(\Sigma)\right)}
$$

where the kernel of the operator $R_{j, 0,0}, \underline{K}\left(Z, Z^{\prime}\right)$ satisfies

$$
\begin{aligned}
& \underline{K}\left(Z, Z^{\prime}\right)=\chi(Z) \widetilde{\chi}\left(Z^{\prime}\right) K_{j}\left(2^{-j} z, 2^{-j} z^{\prime}\right) \\
& =e^{i \lambda d\left(2^{-j} Z, 2^{-j} Z^{\prime}\right)} \chi(Z) \chi\left(Z^{\prime}\right) \frac{\tilde{\chi}\left(Z-Z^{\prime}\right)}{\left(\lambda d\left(2^{-j} Z, 2^{-j} Z^{\prime}\right)\right)^{\frac{d-1}{2}}} a_{0}\left(2^{-j} Z, 2^{-j} Z^{\prime}\right) \\
& \quad=\left(\frac{2^{j}}{\lambda}\right)^{\frac{d-1}{2}} e^{i \lambda 2^{-j} d_{j}\left(Z, Z^{\prime}\right)} \chi(Z) \chi\left(Z^{\prime}\right) \frac{\widetilde{\chi}\left(Z-Z^{\prime}\right)}{d_{j}\left(Z, Z^{\prime}\right)^{\frac{d-1}{2}}} a_{0}\left(2^{-j} Z, 2^{-j} Z^{\prime}\right)
\end{aligned}
$$

where $d_{j}\left(Z, Z^{\prime}\right)=2^{j} d\left(2^{-j} Z, 2^{-j} Z^{\prime}\right)$ is the distance (measured in $\mathbb{R}^{d}$ ) between the points $(Z, 0)$ and $\left(Z^{\prime}, 0\right)$ for the family of metrics $g_{m, n}^{j}(X)=g_{m, n}\left(2^{-j} X\right)$ which converges as $j$ tend to the infinity to the metric $I d$ (in the $C^{\infty}$ topology). As a consequence (and using that on the support of the r.h.s., $\frac{1}{2} \leq\left\|Z-Z^{\prime}\right\| \leq 2$ ), our kernel has the following form

$$
\underline{K}\left(Z, Z^{\prime}\right)=\left(\frac{2^{j}}{\lambda}\right)^{\frac{d-1}{2}} e^{i \lambda 2^{-j} d_{j}\left(Z, Z^{\prime}\right)} \sigma\left(Z, Z^{\prime}, j\right)
$$

with $d_{j}\left(Z, Z^{\prime}\right)$ arbitrarily close (for large $j$ ) in $C^{\infty}$ topology to $\left\|Z-Z^{\prime}\right\|$ and $\sigma$ a function uniformly bounded with respect to $j$ in $C_{0}^{\infty}$ topology. Proposition 6.3 is now a consequence of the following non degeneracy property:

Proposition 6.4. - Consider an operator $T$ on $L^{2}\left(\mathbb{R}^{k}\right)$, whose kernel $K\left(Z, Z^{\prime}\right)$ has the following form

$$
K\left(Z, Z^{\prime}\right)=e^{i \mu d_{j}\left(Z, Z^{\prime}\right)} \sigma\left(Z, Z^{\prime}, j\right)
$$

with $d_{j}\left(Z, Z^{\prime}\right)$ arbitrarily close (for large $j$ ) in $C^{\infty}$ topology to $d_{\infty}\left(Z, Z^{\prime}\right)=\left\|Z-Z^{\prime}\right\|$ and $\sigma$ a function uniformly bounded with respect to $j$ in $C^{\infty}$ topology supported in the set

$$
\left\{\left(Z, Z^{\prime}\right) ;\|Z\| \leq 1, \frac{1}{2} \leq\left\|Z-Z^{\prime}\right\| \leq 2\right\} .
$$

Then, for large $j$, it satisfies the bound

$$
\|T\|_{\mathcal{L}\left(L^{2}\left(\mathbb{R}^{k}\right)\right)} \leq C \mu^{-\frac{k-1}{2}}
$$


The proof of this result (for $j=\infty$ ) is standard. We shall recall it to check that it goes through with the parameter $j$. By using partition of unities, we can assume that $\sigma$ is supported in the set

$$
\left\{\left(Z, Z^{\prime}\right) ;\left\|Z-Z_{0}\right\| \leq \epsilon,\left\|Z^{\prime}-Z_{0}^{\prime}\right\| \leq \epsilon,\left\|Z_{0}-Z_{0}^{\prime}\right\| \in\left[\frac{1}{2}, 2\right]\right\}
$$

We take polar coordinates centered in $Z_{0}$ and write $Z^{\prime}=Z_{0}+r \theta$. We can assume that $Z_{0}^{\prime}=\left(r_{0}, 0,0\right)$. We have

$$
\left.\nabla_{Z}\left(\left\|Z-Z^{\prime}\right\|\right)\right|_{Z=Z_{0}}=-\theta
$$

As a consequence, taking as coordinates $Z_{1}, X=\left(Z_{2}, Z_{3}\right)$, we have

$$
\left.\nabla_{X, \theta}^{2}\left(\left\|Z-Z^{\prime}\right\|\right)\right|_{Z=Z_{0}, Z^{\prime}=Z_{0}^{\prime}}=-\mathrm{Id}
$$

and we can apply to the operator $T_{Z_{1}, r}$ obtained by freezing the $T$ and $r$ variables the following classical non-degenerate phase property (see for example [21, Proposition IX.1.1])

Lemma 6.5. - Consider an operator

$$
T_{\lambda} f(\xi)=\int_{\mathbb{R}^{n}} e^{i \lambda \Phi(x, \xi)} \Psi(x, \xi) f(x) d x
$$

where $\Psi(x, \xi)$ is a fixed smooth function of compact support in $\mathbb{R}^{n}$ and the phase $\Phi$ is real valued, smooth and satisfies

$$
\operatorname{det}\left(\frac{\partial^{2} \Phi(x, \xi)}{\partial x_{i} \partial \xi_{j}}\right) \neq 0
$$

Then

$$
\left\|T_{\lambda} f\right\|_{L^{2}\left(\mathbb{R}^{n}\right)} \leq C \lambda^{-n / 2}\|f\|_{L^{2}\left(\mathbb{R}^{n}\right)} .
$$

A simple use of Minkovski inequality gives Proposition 6.4 (in the case $j=+\infty$ ). To conclude the proof for large $j$, we remark that Lemma 6.5 is stable by small (smooth) perturbations.

6.2. Optimality of Theorem 3, - The first regime to take into account for the optimality is the zonal regime. If we consider functions on $\mathbb{S}^{d}$ depending only on the geodesic distance to a fixed point, we obtain the zonal eigenfunctions on $\mathbb{S}^{d}$. The zonal eigenfunctions can be expressed in terms of zonal spherical harmonics which in their turn can be expressed in terms of the classical Jacobi polynomials (see e.g. 19). In that case we can show that we have a pointwise concentration. If $Z_{n}$ is the $n$-th zonal eigenfunction (with eigenvalue $\lambda^{2}=n(n+d-1)$ )

$$
\left|Z_{n}(x)\right| \approx n^{\frac{d-1}{2}}\left\|Z_{n}\right\|_{L^{2}\left(\mathbb{S}^{d}\right)}, \quad d\left(x, x_{0}\right) \leq \frac{c}{n}
$$


As a consequence, for any submanifold $\Sigma \subset \mathbb{S}^{d}$ if we choose a pole $P \in \Sigma$, and consider the family of corresponding zonal eigenfunctions, $Z_{n}$, we obtain

$$
\left\|\left.Z_{n}\right|_{\Sigma}\right\|_{L^{p}(\Sigma)} \geq c n^{\frac{d-1}{2}-\frac{k}{p}}\left\|Z_{n}\right\|_{L^{2}\left(\mathbb{S}^{d}\right)}, \quad c>0 .
$$

and this shows the optimality of Theorem [3 if $k \leq d-2$ and if $k=d-1$ and $\frac{2 d}{d-1} \leq p \leq+\infty$.

To obtain the optimality of Theorem 3 in the last regime $\left(k=d-1,2 \leq p \leq \frac{2 d}{d-1}\right)$, we turn to the highest weight eigenfunctions $e_{n}=n^{\frac{d-1}{4}}\left(x_{1}+i x_{2}\right)^{n}$ (corresponding to eigenvalues $\left.\lambda^{2}=n(n+d-1)\right)$. In that case, if $\Sigma$ contains the geodesic

$$
\gamma=\left\{x=\left(x_{1}, \ldots, x_{d}\right) ; x_{3}=\cdots=x_{d}=0\right\},
$$

then we obtain

$$
\left\|\left.e_{n}\right|_{\Sigma}\right\|_{L^{p}(\Sigma)} \sim n^{\frac{d-1}{4}-\frac{k-1}{2 p}}\left\|e_{n}\right\|_{L^{2}\left(\mathbb{S}^{d}\right)}
$$

\section{References}

[1] N. Anantharaman. The eigenfunctions of the laplacian do not concentrate on sets of topological entropy. Preprint, 2004.

[2] N. Burq, P. Gérard, and N. Tzvetkov. Multilinear estimates for the Laplace spectral projector on compact manifolds. Comptes rendus de l'académie des sciences, 338(Sér. I):359-364, 2004.

[3] N. Burq, P. Gérard, and N. Tzvetkov. Bilinear eigenfunction estimates and the nonlinear Schrödinger equation on surfaces. Inventiones Mathematicae, 159(1):187 - 223, 2005.

[4] N. Burq, P. Gérard, and N. Tzvetkov. Multilinear eigenfunction estimates and global existence for the three dimensional nonlinear Schrödinger equations. Ann. Sci. École Norm. Sup. (4), 38(2):255-301, 2005.

[5] Y. Colin de Verdière. Ergodicité et foctions propres du laplacien. Comm. Math. Phys., 102:187-214, 1985.

[6] S. Gallot, D. Hulin, and J. Lafontaine. Riemannian geometry. Universitext. SpringerVerlag, Berlin, second edition, 1990.

[7] P. Gérard and E. Leichtnam. Ergodic properties of eigenfunctions for the Dirichlet problem. Duke Mathematical Journal, 71:559-607, 1993.

[8] A. Greenleaf and A. Seeger. Fourier integral operators with fold singularities. J. Reine Angew. Math., 455:35-56, 1994.

[9] B. Helffer, A. Martinez, and D. Robert. Ergodicité et limite semi-classique. Communications in Mathematical Physics, 109:313-326, 1987.

[10] L. Hörmander. The spectral function of an elliptic operator. Acta Math., 121:193-218, 1968. 
[11] L. Hörmander. The Analysis of Linear Partial Differential Operators I, volume 256 of Grundlehren der mathematischen Wissenschaften. Springer Verlag, 1983.

[12] L. Hörmander. The Analysis of Linear Partial Differential Operators IV, volume 275 of Grundlehren der mathematischen Wissenschaften. Springer Verlag, 1985.

[13] Elon Lindenstrauss. Invariant measures and arithmetic quantum unique ergodicity. Ann. of Math. (2), 163(1):165-219, 2006.

[14] R. B. Melrose. Equivalence of glancing hypersurfaces. Invent. Math., 37(3):165-191, 1976.

[15] A. Reznikov. Norms of geodesic restrictions on hyperbolic surfaces and representation theory. Preprint, 2004.

[16] P. Sarnak. Arithmetic quantum chaos. In The Schur lectures (1992) (Tel Aviv), volume 8 of Israel Math. Conf. Proc., pages 183-236. Bar-Ilan Univ., Ramat Gan, 1995.

[17] A.I. Shnirelman. Ergodic properties of eigenfunctions. Uspekhi Mat. Nauk, 29:181-182, 1974.

[18] C. Sogge. Concerning the $L^{p}$ norm of spectral clusters for second order elliptic operators on compact manifolds. Jour. of Funct. Anal., 77:123-138, 1988.

[19] C. Sogge. Fourier integrals in classical analysis. Cambridge tracts in Mathematics, 1993.

[20] C. Sogge and S. Zelditch. Riemannian manifolds with maximal eigenfunction growth. Duke Math. J., 114(3):387-437, 2002.

[21] E. M. Stein. Harmonic analysis: real-variable methods, orthogonality, and oscillatory integrals. Princeton University Press, Princeton, NJ, 1993. With the assistance of Timothy S. Murphy, Monographs in Harmonic Analysis, III.

[22] G. Szegö. Orthogonal polynomials. Colloq. Publications. Amer. Math. Soc., 1974.

[23] D. Tataru. On the regularity of boundary traces for the wave equation. Ann. Scuola Norm. Sup. Pisa Cl. Sci. (4), 26(1):185-206, 1998.

[24] M. Taylor. Diffraction effects in the scattering of waves. In Singularities in boundary value problems (Proc. NATO Adv. Study Inst., Maratea, 1980), volume 65 of NATO Adv. Study Inst. Ser. C: Math. Phys. Sci., pages 271-316. Reidel, Dordrecht, 1981.

[25] S. Zelditch. Uniform distribution of eigenfunctions on compact hyperbolic surfaces. Duke Math. Jour., 55:919-941, 1987.

[26] S. Zelditch and M. Zworski. Ergodicity of eigenfunctions for ergodic billiards. Communications in Mathematical Physics, 175:673-682, 1996.

N. Burq, Université Paris Sud, Mathématiques, Bât 425, 91405 Orsay Cedex et Institut Universitaire de France • E-mail : Nicolas.burq@math.u-psud.fr

P. GÉrARd, Université Paris Sud, Mathématiques, Bât 425, 91405 Orsay Cedex

E-mail : Patrick.gerard@math.u-psud.fr

N. Tzvetkov, Département de Mathématiques, Université Lille I, 59655 Villeneuve d'Ascq Cedex, France • E-mail : Nikolay.tzvetkov@math.univ-lille1.fr 